\title{
Optimal dividends under Erlang(2) inter-dividend decision times
}

\author{
Benjamin Avanzi ${ }^{\mathrm{a}, \mathrm{b}}$, Vincent $\mathrm{Tu}^{\mathrm{a}, *}$, Bernard Wong ${ }^{\mathrm{a}}$ \\ ${ }^{a}$ School of Risk and Actuarial Studies, UNSW Australia Business School \\ UNSW Sydney NSW 2052, Australia \\ ${ }^{b}$ Département de Mathématiques et de Statistique, Université de Montréal \\ Montréal QC H3T 1J4, Canada
}

\begin{abstract}
In the classical dividends problem, dividend decisions are allowed to be made at any time. Under such a framework, the optimal dividend strategies are often of barrier or threshold type, which can lead to very irregular dividend payments over time. In practice however companies distribute dividends on a periodic basis. In that spirit, "Erlangisation" techniques have been used to approximate problems with fixed interdividend decision times.

When studying the optimality of such strategies, the existing literature focuses exclusively on the special case of exponential - that is, Erlang(1) - inter-dividend decision times. Higher dimensional models are surprisingly difficult to study due to the implicit nature of some of the equations. While some of this difficulty continues to exist in high dimensions, in this paper we provide a stepping stone to the general Erlang $(n)$ problem by providing a detailed analysis of the optimality of periodic barrier strategies when inter-dividend-decision times are Erlang(2) distributed. Results are illustrated.
\end{abstract}

Keywords: Brownian motion, Stochastic control, Dividends, Periodic strategies, Barrier strategies, Erlangisation

JEL codes: C44, C61, G24, G32, G35

This paper was accepted for publication in Insurance: Mathematics and Economics

\section{Introduction}

In actuarial risk theory, stochastic processes are used to model the surplus of a company. In the early 20th century, the probability of ruin - the probability that the surplus becomes negative - is used to assess the stability of a company (see, for example, Bühlmann, 1970; Asmussen and Albrecher, 2010). Due to the inverse relationship between probability of ruin and surplus, one should accumulate surplus to infinity in order to minimise the probability ruin. In response to this unrealistic feature, Bruno de Finetti (1957) proposed an alternative formulation of the stability criterion by allowing surplus to be released. Many papers have adopted this criterion and studied it; see Albrecher and Thonhauser (2009) and Avanzi (2009) for reviews.

Often, optimal decisions on when and how much dividends should be paid (i.e. an optimal dividend strategy) are determined by maximising the expected present value of dividends. However Gerber (1974) argued that this pure maximisation may lead to optimal strategies that are usually not realistic. In many surplus models, optimal dividend strategies are of barrier or threshold types. Such strategies typically have too irregular dividend payments, which is arguably unrealistic. Some studies have tried to address Gerber (1974)'s criticism by incorporating real practices adopted by companies (see Avanzi, Tu, and Wong,

\footnotetext{
* Corresponding author.

Email addresses: b.avanzi@unsw.edu.au (Benjamin Avanzi), v.tu@unsw.edu.au (Vincent Tu), bernard.wong@unsw.edu.au (Bernard Wong)
} 
2016, for a discussion of this). Motivated by the fact that companies tend to pay dividends at regular and pre-determined times, periodic dividend strategies (as introduced by Albrecher, Gerber, and Shiu, 2011b) attracted increasing attention among some of the most recent developments.

Because dividends tend to be paid bi-annually or annually, it may be desirable to model dividends distributions at fixed (deterministic) decision times. However this leads to non-Markovian models. An "Erlangisation" technique was proposed by Asmussen, Avram, and Usabel (2002) to approximate finitetime ruin problems. Albrecher, Cheung, and Thonhauser (2011a) applied this technique to study periodic dividend problems in the Cramér-Lundberg model. There, Erlang $(n)$ inter-decision times are used as an approximate solution to periodic dividend problems with fixed inter-decision times. Avanzi, Cheung, Wong, and Woo (2013) studied the expected present value of dividends under periodic barrier strategies when ruin can happen at any time, in the dual model with diffusion and $\operatorname{Erlang}(n)$ inter-decision times (and ruin that can happen at any time). Choi and Cheung (2014) studied a similar problem in the Cramér-Lundberg model.

In a different light, Albrecher and Thonhauser (2012) applied an Erlangisation treatment to approximate a deterministic ruin time (not dividend decision times). The authors first study a dividend maximisation problem by applying exponential killing, that is, they randomise the deterministic ruin time using an exponential random variable. The authors then extend the solution procedure to an Erlang(2) time horizon.

When determining the optimal periodic dividend strategy, there are typically two major steps. First, one typically proposes a candidate solution (e.g., a barrier or threshold type) and obtains the associated expected present value of dividends until ruin. The next step to derive sufficient analytical properties of the proposed solutions (e.g., boundedness of the function) and to check whether it satisfies conditions of a so-called verification lemma. If one focuses on a Brownian motion model where ruin occurs as soon as the surplus hits zero, then the first step described above was first performed by Avanzi, Cheung, Wong, and Woo (2013), who found implicit solutions of the expected present value of dividends of periodic barrier strategies with $\operatorname{Erlang}(n)$ decision times by solving a system of integro-differential equations. However, explicit solutions of expected present value of dividends with tractable (enough) analytical properties are currently only available for periodic strategies with Erlang(1) inter-decisions times (see, e.g., Albrecher, Gerber, and Shiu, 2011b). This leads to optimality results on periodic dividend strategies only available in the special case of Erlang(1) inter-decision times (i.e. exponential). For example, Wei, Wang, and Yang (2012) studied the optimality of periodic barrier strategies in a regime switching Brownian risk model and Avanzi, Tu, and Wong (2014) studied a similar problem in a dual risk model with diffusion. The optimality of periodic barrier strategies is further generalised in a spectrally positive Lévy process in Pérez and Yamazaki (2017).

In this paper, we show that periodic dividend barrier strategies are globally optimal when inter-dividenddecision times are Erlang(2) distributed. While some results are presented for any $n$, it turns out that even in the relatively simple cases considered in this paper (i.e., barrier strategies in a pure diffusion with constant coefficients), we still cannot derive fully explicit formulas for quantities such as the expected present value of dividends for periodic barrier strategies with $\operatorname{Erlang}(n)$ inter-decision times for any $n$. This prevents us from obtaining sufficient properties required to verify the global optimality of periodic barrier strategies. However, in the case of Erlang(2) inter-decision times, we propose some new functions that are special linear combinations of exponential functions, which allow us to perform the full analysis.

We first set up the optimal dividend problems in Section 2. We develop a verification lemma and its proof in Section 3. In Sections 4 and 5, we show the optimality of liquidation-at-first-opportunity strategies, and periodic strategies with strictly positive barriers (respectively) when inter-decision times are Erlang(2) distributed. This is done by obtaining explicit solutions for the expected present value of dividends and their associated analytical properties. This allows us to verify all the conditions in the associated verification lemma. Results are illustrated in Section 6. In the Appendix, we further derive semi-explicit solutions for the expected present value of dividends until ruin for arbitrary periodic barrier strategies with $\operatorname{Erlang}(n)$ interdecision times. In so doing, we develop an algorithm which is used in Section 5. This provides interesting insights on the structural patterns of the expected present value of dividends as the dimension of $n$ increases. 


\section{Problem formulation}

\subsection{Erlang(n) Periodic dividend strategies}

The surplus $\{U(t), t \geq 0\}$ follows a Brownian motion,

$$
U(t)=x+\mu t+\sigma W(t),
$$

where $x$ is the initial surplus, $\mu$ is the drift and $\sigma$ is the volatility. The process $\{W(t)\}$ is a standard Brownian motion. We also assume a complete filtered probability space $\left(\Omega, \mathcal{F},\left\{\mathcal{F}_{t}\right\}, \mathbb{P}\right)$. Dividends can be distributed periodically, at time points $\left\{T_{k}\right\}_{k=1}^{\infty}$. In addition, we assume that the times between successive dividend decision times $\left(T_{k}-T_{k-1}\right)$ follow an independent and identically distributed (i.i.d.) $\operatorname{Erlang}(n)$ distribution with the following density

$$
f(t)=\frac{\gamma^{n} t^{n-1} e^{-\gamma t}}{(n-1) !}, \quad t>0,
$$

which is a gamma distribution with integer shape parameter $n \geq 1$ and positive scale parameter $\gamma>0$. The motivation for using an $\operatorname{Erlang}(n)$ random variable is that we can use its properties to approximate any fixed time horizon. By fixing the mean $n / \gamma$ while increasing $n$ and $\gamma$, the variance $n / \gamma^{2}$ decreases and the limiting $\operatorname{Erlang}(n)$ random variable will approximate deterministic inter-dividend decision times. The term "Erlangisation" thus refers to the technique of using Erlang $(n)$ random variables to approximate deterministic time horizons. Note that the optimality results of this paper focus on the case $n=2$. However, both the verification lemma of Section 3 and the expected present value in Appendix A are developed for any $n$. This explains why the rest of this section is still establishing notation for any $n$.

Using the fact that an $\operatorname{Erlang}(n)$ random variable is a sum of $n$ i.i.d. exponential random variables with parameter $\gamma$, we can re-formulate a periodic dividend strategy (with $\operatorname{Erlang}(n)$ inter-dividend-decision times) using a Markov chain. Consider an $n$-state continuous-time Markov chain and the $i$-th state space means that the time until the next dividend decision time from now is distributed as an $\operatorname{Erlang}(i)$ random variable. To keep track the state space, we define $\epsilon(t)$ to represent the state at any time $t \geq 0$ and we make no assumption on the initial state at time zero $\epsilon(0)$. Using the phase-type structure of Erlang random variables, we can write down the strongly irreducible generator associated with the Markov chain

$$
\begin{gathered}
i=1 \\
i=2 \\
\cdots \\
\cdots \\
i=n-1 \\
i=n
\end{gathered}\left[\begin{array}{ccccccc}
-\gamma & 0 & 0 & \cdots & 0 & 0 & \gamma \\
\gamma & -\gamma & 0 & \cdots & 0 & 0 & 0 \\
0 & \gamma & -\gamma & \cdots & 0 & 0 & 0 \\
\cdots & \cdots & \cdots & \cdots & \cdots & \cdots & \cdots \\
0 & 0 & 0 & \cdots & \gamma & -\gamma & 0 \\
0 & 0 & 0 & \cdots & 0 & \gamma & -\gamma
\end{array}\right] .
$$

For states $2,3, \ldots, n$, the Markov chain jumps to the lower state with intensity $\gamma$, moving closer to the next dividend decision time. When the Markov chain is in state 1, then it moves back to state $n$ with intensity $\gamma$ and a dividend decision is made as the transition occurs. Since the intensity parameter in the generator matrix is $\gamma$, then the jumps between states arrive according to a Poisson process $\left\{N_{\gamma}(t)\right\}$ with constant intensity $\gamma$ and we denote the jump times of $\left\{N_{\gamma}(t)\right\}$ as $\mathscr{T}$. Furthermore, in order to keep track the time spent in each state, we define $n$ auxiliary processes

$$
\eta_{i}(t)=1_{\{\epsilon(t)=i\}} \quad \text { for } i=1,2,3, \ldots, n,
$$

which have càdlàg paths and are square integrable processes (see Lemma 9.2 in Lipster and Shiryaev, 1977). A process $\eta_{i}(t)$ takes the value of 1 when the Markov chain is in state $i$ at time $t \geq 0$.

In order to illustrate the relationships between the auxiliary processes and jump times $\mathscr{T}$, we provide an example of the underlying Markov chain associated with a periodic strategy with Erlang(4) inter-decision times in Figure 1. In Figure 1, we first plot (top row) the process $\left\{d N_{\gamma}(t)\right\}$ which takes the value of 1 when there is a jump in the underlying Poisson process and 0 elsewhere. The times where the process $\left\{d N_{\gamma}(t)\right\}$ 


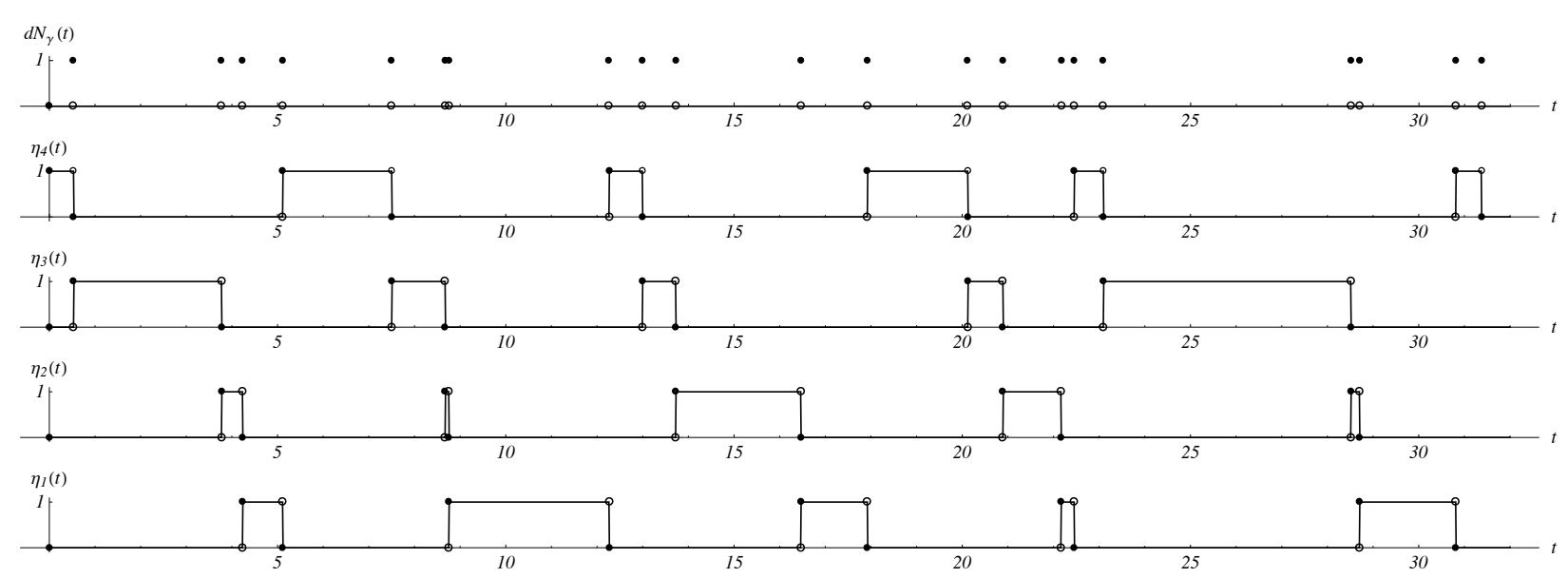

Figure 1: One set of sample paths of $d N_{\gamma}(t), \eta_{4}(t), \eta_{3}(t), \ldots, \eta_{1}(t)$ where $\epsilon(0)=4$

takes the value of 1 represent one realisation of the set $\mathscr{T}$. We then plot $\left\{\eta_{i}(t)\right\}_{i=1}^{4}$ (subsequent rows) and observe that $\eta_{4}(t)+\eta_{3}(t)+\eta_{2}(t)+\eta_{1}(t)=1$ for $t \geq 0$ since time is partitioned into sub-periods spent in each state.

In light of Figure 1, we can partition the set of jump times $\mathscr{T}$ into $n$ disjoint sets of jump times $\mathscr{D}_{1}$, $\mathscr{D}_{2}, \ldots, \mathscr{D}_{n-1}$ and $\mathscr{D}_{n}$,

$$
\mathscr{T}=\bigcup_{i=1}^{n} \mathscr{D}_{i} \quad \text { and } \quad \mathscr{D}_{i} \cap \mathscr{D}_{j}=\emptyset \quad \text { for } i, j=1,2, \ldots, n .
$$

Here the set $\mathscr{D}_{i}$ represents the time points of entering state $i$, which can be further defined to be the common jump times of the process $\left\{\eta_{i}(t)\right\}$ and $\left\{d N_{\gamma}(t)\right\}$, i.e. $\left\{\eta_{i}(t) \cdot d N_{\gamma}(t)\right\}$ for $i=1,2, \ldots, n$ (see Figure 1 for an example). Using this definition, the set of times when the Markov chain enters state $n$ (i.e. $\mathscr{D}_{n}$ ) represents the set of dividend decision times.

Dividends are distributed from the surplus according to a periodic strategy at each dividend decision time and the surplus after dividend distributions is defined as

$$
X(t)=x+\mu t+\sigma W(t)-D(t),
$$

where $\{D(t), t \geq 0\}$ is the aggregate dividends process with $D(0)=0$. We denote the dividends paid at time $t$ as $\left\{\vartheta_{t}, t \geq 0\right\}$. We can use the auxiliary process $\eta_{n}(t)$ to write the aggregate dividends process associated with an $\operatorname{Erlang}(n)$ periodic strategy as

$$
D(t)=\int_{0}^{t} \vartheta_{s} \eta_{n}(s) d N_{\gamma}(s)
$$

Assuming that the time until the next dividend decision is $\operatorname{Erlang}(i)$ distributed, the expected present value of dividends paid until ruin under a periodic strategy $\Theta$ with $\operatorname{Erlang}(n)$ inter-decision times is defined by the following conditional expectation

$$
J(x, i ; \Theta)=\mathbb{E}_{x, i}\left[\int_{0}^{\tau} e^{-\delta s} \vartheta_{s} \eta_{n}(s) d N_{\gamma}(s)\right], \quad x \geq 0, \quad i=1,2, \ldots, n-1, n,
$$

where $\delta$ represents the time preference of investors, and where $\mathbb{E}_{x, i}$ is the conditional expectation given the initial surplus $X(0)=x$ and initial state space $\epsilon(0)=i$.

\subsection{Definition of admissible and optimal periodic dividend strategies}

Let $\mathcal{D}$ denote the set of admissible periodic strategies with $\operatorname{Erlang}(n)$ inter-decision times. To be admissible, a strategy $\Theta \in \mathcal{D}$ has an associated aggregate dividend process $\{D(t)\}$ that is a non-decreasing and 
$\mathcal{F}_{t}$-adapted with càdlàg sample paths and with initial value $D(0)=0$. Jump times associated with $\mathscr{T}, \mathscr{D}_{1}$, $\mathscr{D}_{2}, \ldots, \mathscr{D}_{n}$ are stopping times with respect to $\left\{\mathcal{F}_{t}\right\}$. Dividend decision times correspond to the jump times of $\mathscr{D}_{n}$ are denoted as $\left\{T_{1}, T_{2}, \ldots\right\}$ and satisfy

$$
0<T_{1}<T_{2}<\ldots \quad \text { a.s. }
$$

The amount of dividends paid at decision time $T_{j}$ is $\vartheta_{T_{j}}$ for $j=1,2, \ldots$, which is measurable with respect to $\left\{F_{T_{j}}\right\}$. Dividends cannot exceed the amount of surplus that is currently available, that is,

$$
0 \leq D\left(T_{j}\right)-D\left(T_{j}-\right)=\vartheta_{T_{j}} \leq X\left(T_{j}\right) \text { for } j=1,2, \ldots
$$

For an admissible periodic dividend strategy, the expected present value of dividend with initial surplus $x$ is defined in (2.7). Let $V(x, i)$ be the expected present value of dividends associated with the optimal periodic dividend strategy with $\operatorname{Erlang}(n)$ inter-decision times given the time until next dividend decision is $\operatorname{Erlang}(i)$ distributed, i.e.,

$$
V(x, i)=\sup _{\Theta \in \mathcal{D}} J(x, i ; \Theta), \quad i=1,2, \ldots, n,
$$

where $\mathcal{D}$ denotes the set of all admissible periodic strategies. We qualify a strategy as optimal with dividend payments $\Theta^{*}=\left\{\vartheta_{T_{1}}^{*}, \vartheta_{T_{2}}^{*}, \vartheta_{T_{3}}^{*}, \ldots\right\}$ if for all $i=1,2, \ldots, n$,

$$
V(x, i)=J\left(x, i ; \Theta^{*}\right)=\mathbb{E}_{x, i}\left[\int_{0}^{\tau} e^{-\delta s} \vartheta_{s}^{*} \eta_{n}(s) d N_{\gamma}(s)\right], \quad x \geq 0 .
$$

In the remaining part of the paper, we consider a subclass of periodic dividend strategies - periodic barrier strategies. In order for a periodic barrier strategy to be admissible, the barrier levels can either be strictly positive or zero (the case of zero barrier is also known as a 'liquidation-at-first-opportunity' strategy, which is studied in Section 4) . Therefore when showing the global optimality of periodic barrier strategies, one has to consider both cases. In this paper, we first determine conditions where a liquidation-at-firstopportunity strategy is optimal (with the help of our verification lemma) in Section 4. This then allows us to focus on periodic barrier strategies with strictly positive barriers in Section 5. Unfortunately due to lack of explicit enough solutions, we are unable to show that the two former cases that are considered (null optimal barrier, and positive optimal barrier) are perfect complements, although they are clearly disjoint; see also Section 6.3 .

\subsection{Ruin}

The ruin time $\tau$ is defined as

$$
\tau=\inf \{t \geq 0: X(t)=0\},
$$

that is, ruin is assumed to occur as soon the surplus is depleted. In the remainder of this section, we show that ultimate ruin is certain for periodic barrier strategies with $\operatorname{Erlang}(n)$ inter-decision times. This result is later used in Section 5.3 to verify the optimality of periodic barrier strategies with Erlang(2) inter-decision times.

Suppose that we follow a periodic barrier strategy (barrier level $b>0$ ) with $\operatorname{Erlang}(n)$ inter-decision times. The probability of ultimate ruin is denoted as

$$
\psi_{b}(x, i)=\mathbb{P}_{x, i}[\tau<\infty], \quad \text { for } i=1,2,3, \ldots, n,
$$

where $\tau$ is defined in (2.12) and where $\mathbb{P}_{x, i}$ is the conditional probability given the initial surplus is $X(0)=x$ and initial Erlang clock is $\epsilon(0)=i$. Next we show that $\psi_{b}(x, 1)=\psi_{b}(x, 2)=\ldots=\psi_{b}(x, n)=1$.

The probability of never ruin can be expressed as the product

$$
1-\psi_{b}(x, i)=\mathbb{P}_{x, i}\left[\inf _{0 \leq s<T_{1}} X(s)>0 \mid x>0\right] \cdot \prod_{k=1}^{\infty} \mathbb{P}_{x, n}\left[\inf _{T_{k} \leq s<T_{k+1}} X(s)>0 \mid X\left(T_{k}\right)>0\right],
$$


for $i=1,2,3, \ldots, n$, and where $T_{1}, T_{2}, \ldots$ are the dividend decision times defined in Section 2.2. Since the modified surplus process starts at a level no larger than $b$ at each dividend decision time, we can construct the following inequality

$$
\mathbb{P}_{x, n}\left[\inf _{T_{k} \leq s<T_{k+1}} X(s)>0 \mid X\left(T_{k}\right)>0\right] \leq \mathbb{P}_{x, n}\left[\inf _{T_{k} \leq s<T_{k+1}} X(s)>0 \mid X\left(T_{k}\right)=b\right]:=p
$$

for $k=1,2,3, \ldots$ and clearly $p$ is strictly less than 1 . Substituting (2.15) into (2.14), this yields

$$
1-\psi_{b}(x, i) \leq \mathbb{P}_{x, i}\left[\inf _{0 \leq s<T_{1}} X(s)>0 \mid x>0\right] \lim _{k \rightarrow \infty} p^{k}=0 .
$$

This completes the proof $\psi_{b}(x, i)=1$ for $i=1,2,3, \ldots, n$.

\section{Verification Lemma}

In this section, we present a verification lemma that contains the necessary conditions for an $\operatorname{Erlang}(n)$ periodic dividend strategy to be optimal. In order to construct a proof for the verification lemma, we keep track of the changing state space through time (i.e. the distribution of time until the next dividend decision) to prove the verification lemma. We use an approach that is similar to solving optimal dividend problems when the surplus process involves regime switching dynamics. Our method differs in two main ways. Firstly the changing state space does not influence the dynamics of the surplus and secondly dividends are paid when the state space changes from 1 to $n$ (only). This means that existing verification lemmas must be modified to suit our context.

Note that the lemma and its proof are essentially the same for $n=2$ or $n>2$; as a consequence, we formulate it for any $n$.

Lemma 3.1. Suppose that we follow a periodic dividend strategy $\left\{\xi_{t}\right\}$ where dividend decision times occur in the set $\mathscr{D}_{n}$. For non-negative functions $\{H(x, j)\}_{j=1}^{n}$ that are bounded above by linear functions and twice continuously differentiable except at countably many points, and satisfy

1. $(\mathscr{A}-\delta-\gamma) H(x, j)+\gamma H(x, j-1)=0$, for $j=2,3, \ldots, n$,

2. $(\mathscr{A}-\delta-\gamma) H(x, 1)+\gamma \max _{0 \leq l \leq x}(l+H(x-l, n)) \leq 0$; and

3. $H(0, j)=0$,

4. $0 \leq H^{\prime}(x, j)<\infty$,

5. $H^{\prime \prime}(x, j) \leq 0$,

for $j=1,2, \ldots, n$ and some constant $l$. The differential operator $\mathscr{A}$ is acting on a twice continuously differentiable function,

$$
\mathscr{A} f(x)=\frac{\sigma^{2}}{2} f^{\prime \prime}(x)+\mu f^{\prime}(x)
$$

We then have

$$
H(x, j) \geq V(x, j),
$$

where $V(x, j)$ is the function defined in $(2.11)$ for $j=1,2, \ldots, n$.

Proof. We first consider $e^{-\delta(t \wedge \tau)} H(X(t \wedge \tau), \epsilon(t \wedge \tau))$ and expand it by considering all possible state spaces of $\epsilon(t \wedge \tau)$ using the definition of $\eta_{j}(t)=1_{\{\epsilon(t)=j\}}$ for $j=1,2, \ldots, n$,

$$
e^{-\delta(t \wedge \tau)} H(X(t \wedge \tau), \epsilon(t \wedge \tau))=\sum_{i=1}^{n} e^{-\delta(t \wedge \tau)} H(X(t \wedge \tau), i) \eta_{i}(t \wedge \tau)
$$


Applying Itô's formula to (3.3), we have

$$
\begin{aligned}
& \sum_{i=1}^{n} e^{-\delta(t \wedge \tau)} H(X(t \wedge \tau), i) \eta_{i}(t \wedge \tau) \\
& =\sum_{i=1}^{n} H(x, i) \eta_{i}(0)+\sum_{i=1}^{n} \int_{0}^{t \wedge \tau} e^{-\delta s}(\mathscr{A}-\delta) H(X(s-), i) \eta_{i}(s-) d s+\sum_{i=1}^{n} \int_{0}^{t \wedge \tau} \sigma e^{-\delta s} H^{\prime}(X(s-), i) \eta_{i}(s) d W(s) \\
& +\sum_{i=1}^{n-1} \sum_{s \in \mathscr{D}_{i}, s \leq t \wedge \tau} e^{-\delta s}\left[H(X(s), i) \eta_{i}(s)-H(X(s-), i) \eta_{i}(s-)\right] \\
& +\sum_{s \in \mathscr{D}_{n}, s \leq t \wedge \tau} e^{-\delta s}\left[H(X(s), n) \eta_{n}(s)-H(X(s-), n) \eta_{n}(s-)\right] \\
& +\sum_{i=2}^{n} \sum_{s \in \mathscr{D}_{i-1}, s \leq t \wedge \tau} e^{-\delta s}\left[H(X(s), i) \eta_{i}(s)-H(X(s-), i) \eta_{i}(s-)\right] \\
& +\sum_{s \in \mathscr{D}_{n}, s \leq t \wedge \tau} e^{-\delta s}\left[H(X(s), 1) \eta_{1}(s)-H(X(s-), 1) \eta_{1}(s-)\right]
\end{aligned}
$$

where the sets of jump times $\mathscr{D}_{k}$ represent the time where the underlying Markov chain enters state $k$, for $k=1,2, \ldots, n-1, n$ (see definitions in Section 2). Here (3.4) and (3.5) reflect positive jumps induced by the processes $\left\{\eta_{k}(t)\right\}$, which all occur when the underlying Markov chain enters state $k$ (i.e. $\mathscr{D}_{k}$ ), for $k=1,2, \ldots, n$. In a similar fashion, (3.6) represents negative jumps induced by the processes $\left\{\eta_{k}(t)\right\}$, which occur when the underlying Markov chain leaves state $k$ (i.e. $\mathscr{D}_{k-1}$, same times as entering state $k-1$ ), for $k=2, \ldots, n$. The component in (3.7) represents negative jumps induced by the processes $\left\{\eta_{1}(t)\right\}$, which (by a similar logic) occurs when the underlying Markov chain leaves state 1 or enters state $n$ (i.e. $\mathscr{D}_{n}$ ).

We now convert these positive and negative random jumps into stochastic integrals with respect to the Poisson process $\left\{N_{\gamma}(t)\right\}$, which also governs the transitions of the underlying Markov chain. We first focus on the positive jumps in (3.4) and (3.5). For each term in the first summation (3.4), we fix one particular $i$ from $i=1,2, \ldots, n-1$,

$$
\begin{aligned}
\sum_{s \in \mathscr{D}_{i}, s \leq t \wedge \tau} e^{-\delta s}\left[H(X(s), i) \eta_{i}(s)-H(X(s-), i) \eta_{i}(s-)\right] & =\sum_{s \in \mathscr{D}_{i}, s \leq t \wedge \tau} e^{-\delta s} H(X(s), i) \eta_{i}(s) \\
& =\sum_{s \in \mathscr{D}_{i}, s \leq t \wedge \tau} e^{-\delta s} H(X(s-), i) \eta_{i+1}(s-) \\
& =\sum_{s \in \mathscr{T}, s \leq t \wedge \tau} e^{-\delta s} H(X(s-), i) \eta_{i+1}(s-) \\
& =\int_{0}^{t \wedge \tau} e^{-\delta s} H(X(s-), i) \eta_{i+1}(s-) d N_{\gamma}(s) .
\end{aligned}
$$

Here (3.8) holds because $\eta_{i}(s)=0$ when time $s$ is right before the Markov chain enters state $i\left(\right.$ i.e. $\eta_{i}(s-)=0$ for $\left.s \in \mathscr{D}_{i}\right)$. Next, $(3.9)$ is firstly due to the càdlàg nature of $X(s)$ (i.e. $X(s)=X(s-)$ ) and secondly because the Markov chain is in state $i+1$ right before entering state $i$ (i.e. $\eta_{i}(s)=\eta_{i+1}(s-)$ for $s \in \mathscr{D}_{i}$ ). Finally we have (3.10) because $\eta_{i}(s)=0$ at those times where transitions are into states other than $i$ (i.e. $\eta_{i}(s)=0$ for $\left.s \in \mathscr{T} \backslash \mathscr{D}_{i}\right)$.

Since positive jumps in $\eta_{n}(t)$ correspond to the times when the chain enters state $n$ (i.e. $\mathscr{D}_{n}$ ), these are 
dividend decision times and we can therefore write (3.5) in the following way,

$$
\begin{aligned}
\sum_{s \in \mathscr{D}_{n}, s \leq t \wedge \tau} e^{-\delta s}\left[H(X(s), n) \eta_{n}(s)-H(X(s-), n) \eta_{n}(s-)\right] & =\sum_{s \in \mathscr{D}_{n}, s \leq t \wedge \tau} e^{-\delta s} H(X(s), n) \eta_{n}(s) \\
& =\sum_{s \in \mathscr{D}_{n}, s \leq t \wedge \tau} e^{-\delta s} H\left(X(s-)-\xi_{s}, n\right) \eta_{1}(s-) \\
& =\sum_{s \in \mathscr{T}, s \leq t \wedge \tau} e^{-\delta s} H\left(X(s-)-\xi_{s}, n\right) \eta_{1}(s-) \\
& =\int_{0}^{t \wedge \tau} e^{-\delta s} H\left(X(s-)-\xi_{s}, n\right) \eta_{1}(s-) d N_{\gamma}(s) .
\end{aligned}
$$

Here we have (3.12) because the process $\eta_{n}(s)$ takes value zero right before the underlying Markov chain enters state $n\left(\eta_{n}(s-)=0\right.$ for $\left.s \in \mathscr{D}_{n}\right)$. Then this leads to (3.13) because firstly a dividend payment reduces the surplus at time $s \in \mathscr{D}_{n}\left(X(s)=X(s-)-\xi_{s}\right)$ and secondly because the Markov chain is in state 1 right before entering state $n$ (i.e. $\eta_{n}(s)=\eta_{1}(s-)$ for $\left.s \in \mathscr{D}_{n}\right)$. Lastly we have (3.14) because $\eta_{n}(s)=0$ at those times where transitions are into states other than $n$ (i.e. $s \in \mathscr{T} \backslash \mathscr{D}_{n}$ ).

Now we move to the negative jumps described in (3.6) and (3.7). We first fix the index $i$ in the first summation of (3.6) for $i=2,3, \ldots, n$, then write

$$
\begin{aligned}
\sum_{s \in \mathscr{D}_{i-1}, s \leq t \wedge \tau} e^{-\delta s}\left[H(X(s), i) \eta_{i}(s)-H(X(s-), i) \eta_{i}(s-)\right] & =-\sum_{s \in \mathscr{D}_{i-1}, s \leq t \wedge \tau} e^{-\delta s} H(X(s-), i) \eta_{i}(s-) \\
& =-\sum_{s \in \mathscr{T}, s \leq t \wedge \tau} e^{-\delta s} H(X(s-), i) \eta_{i}(s-) \\
& =-\int_{0}^{t \wedge \tau} e^{-\delta s} H(X(s-), i) \eta_{i}(s-) d N_{\gamma}(s) .
\end{aligned}
$$

Justifications are similar to those provided along with the equations associated with positive jumps. Here we have (3.16) because the process $\eta_{i}(s)$ is zero when the Markov chain leaves state $i$ (i.e. $\eta_{i}(s)=0$ for $\left.s \in \mathscr{D}_{i-1}\right)$. Next (3.17) is due to the fact that $\eta_{i}(s-)=0$ at times when the Markov chain enters states other than $i-1\left(s \in \mathscr{T} \backslash \mathscr{D}_{i-1}\right)$.

Finally, we consider negative jumps in (3.7), and rewrite

$$
\begin{aligned}
\sum_{s \in \mathscr{D}_{n}, s \leq t \wedge \tau} e^{-\delta s}\left[H(X(s), 1) \eta_{1}(s)-H(X(s-), 1) \eta_{1}(s-)\right] & =-\sum_{s \in \mathscr{D}_{n}, s \leq t \wedge \tau} e^{-\delta s} H(X(s-), 1) \eta_{1}(s-) \\
& =-\sum_{s \in \mathscr{T}, s \leq t \wedge \tau} e^{-\delta s} H(X(s-), 1) \eta_{1}(s-) \\
& =-\int_{0}^{t \wedge \tau} e^{-\delta s} H(X(s-), 1) \eta_{1}(s-) d N_{\gamma}(s) .
\end{aligned}
$$

In a similar fashion, we have (3.19) because $\eta_{1}(s)$ is zero when the Markov chain enters state $n$ (i.e. $\eta_{1}(s)=0$ for $\left.s \in \mathscr{D}_{n}\right)$. Next (3.20) is due to the fact $\eta_{1}(s-)=0$ at times when the Markov chain enters states other than $n$ (i.e. $\eta_{1}(s-)=0$ for $s \in \mathscr{T} \backslash \mathscr{D}_{n}$ ). 
Now substituting (3.11), (3.15), (3.18) and (3.21) into (3.4)-(3.7) respectively yields

$$
\begin{aligned}
& \sum_{i=1}^{n} e^{-\delta(t \wedge \tau)} H(X(t \wedge \tau), i) \eta_{i}(t \wedge \tau) \\
& =\sum_{i=1}^{n} H(x, i) \eta_{i}(0)+\sum_{i=1}^{n} \int_{0}^{t \wedge \tau} e^{-\delta s}(\mathscr{A}-\delta) H(X(s-), i) \eta_{i}(s-) d s+\sum_{i=1}^{n} \int_{0}^{t \wedge \tau} \sigma e^{-\delta s} H^{\prime}(X(s-), i) \eta_{i}(s) d W(s) \\
& +\sum_{i=1}^{n-1} \int_{0}^{t \wedge \tau} e^{-\delta s} H(X(s-), i) \eta_{i+1}(s-) d N_{\gamma}(s)-\sum_{i=2}^{n} \int_{0}^{t \wedge \tau} e^{-\delta s} H(X(s-), i) \eta_{i}(s-) d N_{\gamma}(s) \\
& +\int_{0}^{t \wedge \tau} e^{-\delta s} H\left(X(s-)-\xi_{s}, n\right) \eta_{1}(s-) d N_{\gamma}(s)-\int_{0}^{t \wedge \tau} e^{-\delta s} H(X(s-), 1) \eta_{1}(s-) d N_{\gamma}(s)
\end{aligned}
$$

Since we assume that the process can start in any state $j=1,2, \ldots, n$, we have $\eta_{j}(0)=1$ for and $\eta_{k}(0)=0$ for $k \neq j$. Next we can then combine the integrals in (3.22) by adjusting the summation index and combine the integrals in (3.23), which yields

$$
\begin{aligned}
& e^{-\delta(t \wedge \tau)} H(X(t \wedge \tau), \epsilon(t \wedge \tau))=H(x, j) \\
& +\sum_{i=1}^{n} \int_{0}^{t \wedge \tau} e^{-\delta s}(\mathscr{A}-\delta) H(X(s-), i) \eta_{i}(s-) d s+\sum_{i=1}^{n} \int_{0}^{t \wedge \tau} \sigma e^{-\delta s} H^{\prime}(X(s-), i) \eta_{i}(s) d W(s) \\
& +\sum_{i=2}^{n} \int_{0}^{t \wedge \tau} e^{-\delta s}(H(X(s-), i-1)-H(X(s-), i)) \eta_{i}(s-) d N_{\gamma}(s) \\
& +\int_{0}^{t \wedge \tau} e^{-\delta s}\left(\xi_{s}+H\left(X(s-)-\xi_{s}, n\right)-H(X(s-), 1)\right) \eta_{1}(s-) d N_{\gamma}(s) \\
& -\int_{0}^{t \wedge \tau} e^{-\delta s} \xi_{s} \eta_{n}(s) d N_{\gamma}(s) .
\end{aligned}
$$

Note that we add and subtract the dividend term $\int_{0}^{t \wedge \tau} e^{-\delta s} \xi_{s} \eta_{n}(s) d N_{\gamma}(s)$ to (3.23). Next we change the integrals in (3.24) and (3.25) to stochastic integrals with respect to the compensated Poisson process $\tilde{N}_{\gamma}(t)=N_{\gamma}(t)-\gamma t$,

$$
\begin{aligned}
& e^{-\delta(t \wedge \tau)} H(X(t \wedge \tau), \epsilon(t \wedge \tau))=H(x, j) \\
& +\sum_{i=1}^{n} \int_{0}^{t \wedge \tau} \sigma e^{-\delta s} H^{\prime}(X(s-), i) \eta_{i}(s) d W(s) \\
& +\sum_{i=2}^{n} \int_{0}^{t \wedge \tau} e^{-\delta s}[(\mathscr{A}-\delta-\gamma) H(X(s-), i)+\gamma H(X(s-), i-1)] \eta_{i}(s-) d s \\
& +\sum_{i=2}^{n} \int_{0}^{t \wedge \tau} e^{-\delta s}(H(X(s-), i-1)-H(X(s-), i)) \eta_{i}(s-) d \tilde{N}_{\gamma}(s) \\
& +\int_{0}^{t \wedge \tau} e^{-\delta s}\left[(\mathscr{A}-\delta-\gamma) H(X(s-), 1)+\gamma\left(\xi_{s}+H\left(X(s-)-\xi_{s}, n\right)\right)\right] \eta_{i}(s) d s \\
& +\int_{0}^{t \wedge \tau} e^{-\delta s}\left(\xi_{s}+H\left(X(s-)-\xi_{s}, n\right)-H(X(s-), 1)\right) \eta_{1}(s-) d \tilde{N}_{\gamma}(s) \\
& -\int_{0}^{t \wedge \tau} e^{-\delta s} \xi_{s} \eta_{n}(s) d N_{\gamma}(s) .
\end{aligned}
$$

Using conditions (1) and (2) from the verification lemma, we can simplify (3.27) and (3.29) further and 
obtain the following inequality,

$$
\begin{aligned}
e^{-\delta(t \wedge \tau)} H(X(t \wedge \tau), \epsilon(t \wedge \tau)) \leq & H(x, j)+\sum_{i=1}^{n} \int_{0}^{t \wedge \tau} \sigma e^{-\delta s} H^{\prime}(X(s-), i) \eta_{i}(s) d W(s) \\
& +\sum_{i=2}^{n} \int_{0}^{t \wedge \tau} e^{-\delta s}(H(X(s-), i-1)-H(X(s-), i)) \eta_{i}(s-) d \tilde{N}_{\gamma}(s) \\
& +\int_{0}^{t \wedge \tau} e^{-\delta s}\left(\xi_{s}+H\left(X(s-)-\xi_{s}, n\right)-H(X(s-), 1)\right) \eta_{1}(s-) d \tilde{N}_{\gamma}(s) \\
& -\int_{0}^{t \wedge \tau} e^{-\delta s} \xi_{s} \eta_{n}(s) d N_{\gamma}(s) .
\end{aligned}
$$

Next we take expectations conditionally on the initial surplus and state spaces $\left(\mathbb{E}_{x, j}\right)$, and obtain

$$
\begin{aligned}
\mathbb{E}_{x, j}\left[e^{-\delta(t \wedge \tau)} H(X(t \wedge \tau), \epsilon(t \wedge \tau))\right] \leq & H(x, j) \\
& +\sum_{i=1}^{n} \mathbb{E}_{x, j}\left[\int_{0}^{t \wedge \tau} \sigma e^{-\delta s} H^{\prime}(X(s-), i) \eta_{i}(s) d W(s)\right] \\
& +\sum_{i=2}^{n} \mathbb{E}_{x, j}\left[\int_{0}^{t \wedge \tau} e^{-\delta s}(H(X(s-), i-1)-H(X(s-), i)) \eta_{i}(s-) d \tilde{N}_{\gamma}(s)\right] \\
& +\mathbb{E}_{x, j}\left[\int_{0}^{t \wedge \tau} e^{-\delta s}\left(\xi_{s}+H\left(X(s-)-\xi_{s}, n\right)-H(X(s-), 1)\right) \eta_{1}(s-) d \tilde{N}_{\gamma}(s)\right] \\
& -\mathbb{E}_{x, j}\left[\int_{0}^{t \wedge \tau} e^{-\delta s} \xi_{s} \eta_{n}(s) d N_{\gamma}(s)\right] .
\end{aligned}
$$

Using conditions (4) and (5) from the verification lemma, we can show that stochastic integrals in (3.35), (3.36) and (3.37) are square integrable martingales with zero means and we have the following inequality

$$
\mathbb{E}_{x, j}\left[e^{-\delta(t \wedge \tau)} H(X(t \wedge \tau), \epsilon(t \wedge \tau))\right] \leq H(x, j)-\mathbb{E}_{x, j}\left[\int_{0}^{t \wedge \tau} e^{-\delta s} \xi_{s} \eta_{n}(s) d N_{\gamma}(s)\right] .
$$

By taking the limit of $t \rightarrow \infty$, the RHS of (3.38), we have

$$
\liminf \operatorname{in}_{t \rightarrow \infty} \mathbb{E}_{x, j}\left[e^{-\delta(t \wedge \tau)} H(X(t \wedge \tau), \epsilon(t \wedge \tau))\right] \geq 0 .
$$

Next by the monotone convergence theorem, we obtain

$$
H(x, j) \geq \mathbb{E}_{x, j}\left[\int_{0}^{t \wedge \tau} e^{-\delta s} \xi_{s} \eta_{n}(s) d N_{\gamma}(s)\right]=J(x, j ; \Theta) \Longrightarrow H(x, j) \geq V(x, j) .
$$

Since we assume that the process can start in any state $j$, the proof holds for any $j=1,2, \ldots, n$. This completes the proof of the verification lemma.

In the next two sections, we focus on periodic barrier strategies with Erlang(2) inter-decision times. We first study a liquidation-at-first-opportunity strategy in Section 4. We use the verification lemma and show that a liquidation-at-first-opportunity strategy is optimal under certain conditions. Next we consider periodic strategies with strictly positive and optimal barriers in Section 5 and derive the expected present value of dividends. We then verify the optimality of the periodic barrier strategies after obtaining sufficiently analytical properties. 


\section{Optimal liquidation-at-first-opportunity strategies with $\operatorname{Erlang}(2)$ inter-decision times}

In this section, we use the verification lemma in Section 3 to determine sufficient and necessary conditions for the global optimality of liquidation-at-first-opportunity strategies (periodic barrier strategies with a zero barrier) with Erlang(2) inter-decision times. To avoid potential clash of notation, we denote the expected present value of dividends until ruin as $F(x, i)$, when the next dividend decision has an $\operatorname{Erlang}(i)$ distribution for $i=1$ and 2 . The two functions $F(x, 1)$ and $F(x, 2)$ satisfy the following system of differential equations (see Appendix A.3 for a similar derivation by setting $n=2$ and $b=0$ ) for $x \in[0, \infty)$

$$
\begin{gathered}
\frac{\sigma^{2}}{2} F^{\prime \prime}(x, 2)+\mu F^{\prime}(x, 2)-(\gamma+\delta) F(x, 2)+\gamma F(x, 1)=0 ; \\
\frac{\sigma^{2}}{2} F^{\prime \prime}(x, 1)+\mu F^{\prime}(x, 1)-(\gamma+\delta) F(x, 1)+\gamma x=0,
\end{gathered}
$$

with initial conditions $F(0,1)=F(0,2)=0$. We further assume that both functions approaches some linear functions as $x \rightarrow \infty$. To solve (4.1) and (4.2), we first note that (4.2) is an inhomogeneous second order differential equation for $F(x, 1)$. Using the initial condition and the linear bound assumption, we can determine an explicit solution for $F(x, 1)$

$$
F(x, 1)=\frac{\gamma}{\gamma+\delta}\left(\frac{\mu}{\gamma+\delta}+x\right)-\frac{\mu \gamma}{(\gamma+\delta)^{2}} e^{s_{\gamma} x}
$$

where $r_{\gamma}>0$ and $s_{\gamma}<0$ are roots of the following equation

$$
\frac{\sigma^{2}}{2} \xi^{2}+\mu \xi-(\gamma+\delta)=0
$$

Next we substitute (4.3) back into (4.1) to obtain a general solution for $F(x, 2)$,

$$
F(x, 2)=\left(\frac{\gamma}{\gamma+\delta}\right)^{2}\left(\frac{2 \mu}{\gamma+\delta}+x\right)+A e^{s_{\gamma} x}+B x e^{s_{\gamma} x} .
$$

The initial condition for $F(x, 2)$ can be used to solve for the coefficient $A$

$$
F(0,2)=\left(\frac{\gamma}{\gamma+\delta}\right)^{2} \frac{2 \mu}{\gamma+\delta}+A \Longrightarrow A=-\frac{2 \mu}{\gamma+\delta}\left(\frac{\gamma}{\gamma+\delta}\right)^{2}
$$

Next we can substitute $F(x, 2)$ into $(4.1)$ and obtain the following

$$
\begin{aligned}
F(x, 1) & =-\frac{1}{\gamma}\left(\frac{\sigma^{2}}{2} F^{\prime \prime}(x, 2)+\mu F^{\prime}(x, 2)-(\delta+\gamma) F(x, 2)\right) \\
\Longrightarrow F(x, 1) & =\frac{\gamma}{\gamma+\delta}\left(\frac{\mu}{\gamma+\delta}+x\right)-\frac{\mu+\sigma^{2} s_{\gamma}}{\gamma} B e^{s_{\gamma} x} .
\end{aligned}
$$

Comparing the above equation with (4.3), we have the explicit coefficient $B$

$$
\begin{gathered}
\frac{s_{\gamma}-r_{\gamma}}{r_{\gamma} s_{\gamma}} \frac{\gamma+\delta}{\gamma} B=-\frac{\mu \gamma}{(\gamma+\delta)^{2}} \\
\Longrightarrow B=-\frac{s_{\gamma} r_{\gamma}}{s_{\gamma}-r_{\gamma}} \frac{\mu}{\gamma+\delta}\left(\frac{\gamma}{\gamma+\delta}\right)^{2} .
\end{gathered}
$$

Both functions $F(x, 1)$ and $F(x, 2)$ are now fully specified with explicit form of coefficients and can be shown to be increasing and concave. 
Next we determine the conditions for a liquidation-at-first-opportunity strategy to be globally optimal (i.e. $b^{*}=0$ ). Suppose that such a strategy is optimal, then from condition 2 of the verification lemma we know that an optimal liquidation-at-first-opportunity strategy should satisfy the following inequality

$$
(\mathscr{A}-\delta-\gamma) F(x, 1)+\gamma(l+F(x-l, 2)) \leq 0 .
$$

Substituting $F(x, 1)$ from (4.3) into the above inequality yields

$$
l+F(x-l, 2) \leq x \Longleftrightarrow \frac{F(x-l, 2)-F(0,2)}{x-l} \leq 1,
$$

for $l \in[0, x]$. Now since (4.12) holds true for all $l$ and $F(x, 2)$ is a concave function, we can conclude that $F^{\prime}(x, 2) \leq 1$ for all $x$ and in particular $F^{\prime}(x, 2) \leq 1 \Longleftrightarrow F^{\prime}(0,2) \leq 1$ (due to concavity). Now we can establish that when prospect becomes insufficient such that the inequality $F^{\prime}(0,2) \leq 1$ is satisfied, then the optimal periodic strategy with Erlang(2) inter-decision time is the liquidation-at-first-opportunity strategy (i.e. $\left.b^{*}=0\right)$. We further illustrate different combinations of parameters that lead to the insufficient condition in Section 6.2.

\section{Optimal periodic strategies with Erlang(2) inter-decision times}

In Section 4, we determined conditions where a liquidation-at-first-opportunity strategy is the globally optimal periodic strategy with Erlang(2) inter-decision times. This subsequently allows us to focus on periodic barrier strategies with strictly positive barriers and verify their global optimality with Erlang $(2)$ inter-decision times. In this section, we develop new functions that are special linear combinations of exponential functions. With the help of these new functions, we are able to express the expected present value of dividends in alternative forms and subsequently extract sufficient analytical properties. As we move to higher $n$ in $\operatorname{Erlang}(n)$ inter-decision times, we believe that the methodology can, in principle, be extended. However, this requires substantial work, and is left to future research.

\subsection{Expected present value of dividends until ruin}

We focus on periodic barrier strategies with Erlang(2) inter-decision times when a positive optimal barrier $\left(b^{*}>0\right)$ is applied. Denote the expected present value of dividends until ruin when there are $i$ Erlang clock ticks left as $G\left(x, i ; b^{*}\right)$ for $i=1,2$. For example $G\left(x, 2 ; b^{*}\right)$ represents the expected present value of dividends when the time until the next dividend payment has a distribution of Erlang $(2)$. Next we separate $G\left(x, 2 ; b^{*}\right)$ and $G\left(x, 1 ; b^{*}\right)$ into two branches,

$$
G\left(x, 2 ; b^{*}\right)=\left\{\begin{array}{ll}
G_{L}\left(x, 2 ; b^{*}\right), & x \in\left[0, b^{*}\right) \\
G_{U}\left(x, 2 ; b^{*}\right), & x \in\left[b^{*}, \infty\right)
\end{array} \quad \text { and } \quad G\left(x, 1 ; b^{*}\right)=\left\{\begin{array}{ll}
G_{L}\left(x, 1 ; b^{*}\right), & x \in\left[0, b^{*}\right) \\
G_{U}\left(x, 1 ; b^{*}\right), & x \in\left[b^{*}, \infty\right)
\end{array} .\right.\right.
$$

The two lower branches $G_{L}\left(x, 2 ; b^{*}\right)$ and $G_{L}\left(x, 1 ; b^{*}\right)$ satisfy the following system of differential equations for $x \in\left[0, b^{*}\right)$,

$$
\begin{aligned}
& \frac{\sigma^{2}}{2} G_{L}^{\prime \prime}\left(x, 2 ; b^{*}\right)+\mu G_{L}^{\prime}\left(x, 2 ; b^{*}\right)-(\gamma+\delta) G_{L}\left(x, 2 ; b^{*}\right)+\gamma G_{L}\left(x, 1 ; b^{*}\right)=0 \\
& \frac{\sigma^{2}}{2} G_{L}^{\prime \prime}\left(x, 1 ; b^{*}\right)+\mu G_{L}^{\prime}\left(x, 1 ; b^{*}\right)-(\gamma+\delta) G_{L}\left(x, 1 ; b^{*}\right)+\gamma G_{L}\left(x, 2 ; b^{*}\right)=0,
\end{aligned}
$$

with initial conditions $G_{L}\left(0,2 ; b^{*}\right)=G_{L}\left(0,1 ; b^{*}\right)=0$. First we substitute (5.3) into (5.2) and obtain a forth-order homogeneous differential equation for $G_{L}\left(x, 2 ; b^{*}\right)$

$$
(\mathscr{A}-\delta-\gamma)^{2} G_{L}\left(x, 2 ; b^{*}\right)-\gamma^{2} G_{L}\left(x, 2 ; b^{*}\right)=0 .
$$


The characteristic equation for the forth-order homogeneous differential equation (5.4) is

$$
\left(\frac{\sigma^{2}}{2} \xi^{2}+\mu \xi-(\gamma+\delta)\right)^{2}-\gamma^{2}=0 \Longrightarrow\left(\frac{\sigma^{2}}{2} \xi^{2}+\mu \xi-\delta\right)\left(\frac{\sigma^{2}}{2} \xi^{2}+\mu \xi-(2 \gamma+\delta)\right)=0
$$

which gives roots $r_{0}>0, s_{0}<0$ and $r_{2 \gamma}>0, s_{2 \gamma}<0$. Thus a general solution for $G_{L}\left(x, 2 ; b^{*}\right)$ is

$$
G_{L}\left(x, 2 ; b^{*}\right)=A_{r} e^{r_{0} x}+A_{s} e^{s_{0} x}+B_{r} e^{r_{2 \gamma} x}+B_{s} e^{s_{2 \gamma} x}, \quad x \in\left[0, b^{*}\right),
$$

where $A_{r}, A_{s}, B_{r}$ and $B_{s}$ are constants. Next we find a general solution of $G_{L}\left(x, 1 ; b^{*}\right)$ by substituting the general solution of $G_{L}\left(x, 2 ; b^{*}\right)(5.6)$ into (5.3) and we have

$$
\begin{aligned}
G_{L}\left(x, 1 ; b^{*}\right) & =-\frac{1}{\gamma}(\mathscr{A}-\delta-\gamma) G_{L}\left(x, 2 ; b^{*}\right)=-\frac{1}{\gamma}(\mathscr{A}-\delta-\gamma)\left(A_{r} e^{r_{0} x}+A_{s} e^{s_{0} x}+B_{r} e^{r_{2 \gamma} x}+B_{s} e^{s_{2 \gamma} x}\right) \\
& =A_{r} e^{r_{0} x}+A_{s} e^{s_{0} x}-B_{r} e^{r_{2 \gamma} x}-B_{s} e^{s_{2 \gamma} x}
\end{aligned}
$$

Using initial conditions $G_{L}\left(0,1 ; b^{*}\right)=G_{L}\left(0,2 ; b^{*}\right)=0$, we obtain general solutions for $G_{L}\left(x, 2 ; b^{*}\right)$ and $G_{L}\left(x, 1 ; b^{*}\right)$

$$
\begin{aligned}
& G_{L}\left(x, 2 ; b^{*}\right)=A \cdot h_{0}(x)+B \cdot h_{2 \gamma}(x) ; \\
& G_{L}\left(x, 1 ; b^{*}\right)=A \cdot h_{0}(x)-B \cdot h_{2 \gamma}(x),
\end{aligned}
$$

for $x \in\left[0, b^{*}\right)$, where $A$ and $B$ are constants, $h_{0}(x)=e^{r_{0} x}-e^{s_{0} x}$ and $h_{2 \gamma}(x)=e^{r_{2 \gamma} x}-e^{s_{2 \gamma} x}$.

Next we consider the two upper branches $G_{U}\left(x, 2 ; b^{*}\right)$ and $G_{U}\left(x, 1 ; b^{*}\right)$. They satisfy the following system of differential equations for $x \in\left[b^{*}, \infty\right)$,

$$
\begin{gathered}
\frac{\sigma^{2}}{2} G_{U}^{\prime \prime}\left(x, 2 ; b^{*}\right)+\mu G_{U}^{\prime}\left(x, 2 ; b^{*}\right)-(\gamma+\delta) G_{U}\left(x, 2 ; b^{*}\right)+\gamma G_{U}\left(x, 1 ; b^{*}\right)=0 \\
\frac{\sigma^{2}}{2} G_{U}^{\prime \prime}\left(x, 1 ; b^{*}\right)+\mu G_{U}^{\prime}\left(x, 1 ; b^{*}\right)-(\gamma+\delta) G_{U}\left(x, 1 ; b^{*}\right)+\gamma\left(x-b^{*}+G_{L}\left(b^{*}, 2 ; b^{*}\right)\right)=0 .
\end{gathered}
$$

Using the algorithm developed in Appendix A.3, we obtain general solutions of $G_{U}\left(x, 1 ; b^{*}\right)$ and $G_{U}\left(x, 2 ; b^{*}\right)$

$$
\begin{aligned}
& G_{U}\left(x, 2 ; b^{*}\right)=\left(\frac{\gamma}{\gamma+\delta}\right)^{2}\left[\frac{2 \mu}{\gamma+\delta}+x-b^{*}+G_{L}\left(b^{*}, 2 ; b^{*}\right)\right]+\left(C+D\left(x-b^{*}\right)\right) e^{s_{\gamma}\left(x-b^{*}\right)} \\
& G_{U}\left(x, 1 ; b^{*}\right)=\left(\frac{\gamma}{\gamma+\delta}\right)\left[\frac{\mu}{\gamma+\delta}+x-b^{*}+G_{L}\left(b^{*}, 2 ; b^{*}\right)\right]+D \frac{s_{\gamma}-r_{\gamma}}{s_{\gamma} r_{\gamma}} \frac{\gamma+\delta}{\gamma} e^{s_{\gamma}\left(x-b^{*}\right)}
\end{aligned}
$$

where $C$ and $D$ are constant coefficients. Since $G\left(x, 2 ; b^{*}\right)$ and $G\left(x, 1 ; b^{*}\right)$ are continuous up to the second order differentiation and $G_{L}^{\prime}\left(b^{*}, 2 ; b^{*}\right)=G_{U}^{\prime}\left(b^{*}, 2 ; b^{*}\right)=1$ when the optimal barrier $b^{*}$ is applied, we have

$$
\begin{aligned}
G_{L}^{\prime \prime}\left(b^{*}, 2 ; b^{*}\right)=G_{U}^{\prime \prime}\left(b^{*}, 2 ; b^{*}\right) & \Longrightarrow A \cdot h_{0}^{\prime \prime}\left(b^{*}\right)+B \cdot h_{2 \gamma}^{\prime \prime}\left(b^{*}\right)=2 D s_{\gamma}+C s_{\gamma}^{2} ; \\
G_{L}^{\prime \prime}\left(b^{*}, 1 ; b^{*}\right)=G_{U}^{\prime \prime}\left(b^{*}, 1 ; b^{*}\right) & \Longrightarrow A \cdot h_{0}^{\prime \prime}\left(b^{*}\right)-B \cdot h_{2 \gamma}^{\prime \prime}\left(b^{*}\right)=D \frac{s_{\gamma}-r_{\gamma}}{s_{\gamma} r_{\gamma}} \frac{\gamma+\delta}{\gamma} s_{\gamma}^{2} ; \\
G_{L}^{\prime}\left(b^{*}, 1 ; b^{*}\right)=G_{U}^{\prime}\left(b^{*}, 1 ; b^{*}\right) & \Longrightarrow A \cdot h_{0}^{\prime}\left(b^{*}\right)-B \cdot h_{2 \gamma}^{\prime}\left(b^{*}\right)=\left(\frac{\gamma}{\gamma+\delta}\right)+D \frac{s_{\gamma}-r_{\gamma}}{s_{\gamma} r_{\gamma}} \frac{\gamma}{\gamma} s_{\gamma} ; \\
G_{L}^{\prime}\left(b^{*}, 2 ; b^{*}\right)=1 & \Longrightarrow A \cdot h_{0}^{\prime}\left(b^{*}\right)+B \cdot h_{2 \gamma}^{\prime}\left(b^{*}\right)=1 ; \\
G_{U}^{\prime}\left(b^{*}, 2 ; b^{*}\right)=1 & \Longrightarrow\left(\frac{\gamma}{\gamma+\delta}\right)^{2}+D+C s_{\gamma}=1,
\end{aligned}
$$

which are 5 equations for 5 unknowns $\left(A, B, C, D\right.$ and $\left.b^{*}\right)$. Explicit solutions in (5.8), (5.9), (5.12) and (5.13) can also be obtained using similar methods from Avanzi, Cheung, Wong, and Woo (2013, Sections 3.3 and 3.4 ) by removing jumps from the surplus process. 
Remark 5.1. Note that the conditions $G_{L}^{\prime \prime}\left(b^{*}, i ; b^{*}\right)=G_{U}^{\prime \prime}\left(b^{*}, i ; b^{*}\right)$ and $G_{L}^{\prime}\left(b^{*}, i ; b^{*}\right)=G_{U}^{\prime}\left(b^{*}, i ; b^{*}\right) i m p l y$ that $G_{L}\left(b^{*}, i ; b^{*}\right)=G_{U}\left(b^{*}, i ; b^{*}\right)$ for $i=1,2$. In fact any two of the three equations imply the third, which can be verified by substituting $x=b^{*}$ into (5.12) and (5.13). Here we choose to use the first and second order pairs of conditions for the convenience of solving the coefficients. This is also used in periodic barrier strategies with Erlang(1) inter-decision times (see, e.g., Remark 4.3 in Avanzi, Tu, and Wong, 2014).

We solve (5.14)-(5.17) simultaneously and obtain explicit forms for the coefficients $A, B, C, D$ in terms of $b^{*}$. Here we first assume that a positive optimal barrier $b^{*}$ exists and then later on determine the equation that $b^{*}$ has to satisfy. We have

$$
\begin{aligned}
A & =\frac{\mathcal{H}_{2 \gamma}^{\prime}\left(b^{*}\right)}{h_{0}^{\prime}\left(b^{*}\right) \mathcal{H}_{2 \gamma}^{\prime}\left(b^{*}\right)+h_{2 \gamma}^{\prime}\left(b^{*}\right) \mathcal{H}_{0}^{\prime}\left(b^{*}\right)} ; \\
B & =\frac{\mathcal{H}_{0}^{\prime}\left(b^{*}\right)}{h_{0}^{\prime}\left(b^{*}\right) \mathcal{H}_{2 \gamma}^{\prime}\left(b^{*}\right)+h_{2 \gamma}^{\prime}\left(b^{*}\right) \mathcal{H}_{0}^{\prime}\left(b^{*}\right)} ; \\
C & =\frac{1}{s_{\gamma}^{2}} \frac{h_{0}^{\prime \prime}\left(b^{*}\right) \mathcal{H}_{2 \gamma}^{\prime}\left(b^{*}\right)+h_{2 \gamma}^{\prime \prime}\left(b^{*}\right) \mathcal{H}_{0}^{\prime}\left(b^{*}\right)}{h_{0}^{\prime}\left(b^{*}\right) \mathcal{H}_{2 \gamma}^{\prime}\left(b^{*}\right)+h_{2 \gamma}^{\prime}\left(b^{*}\right) \mathcal{H}_{0}^{\prime}\left(b^{*}\right)}-\frac{r_{\gamma}}{s_{\gamma}-r_{\gamma}} \frac{\gamma}{\gamma+\delta} \frac{2}{s_{\gamma}^{2}} \frac{h_{0}^{\prime \prime}\left(b^{*}\right) \mathcal{H}_{2 \gamma}^{\prime}\left(b^{*}\right)-h_{2 \gamma}^{\prime \prime}\left(b^{*}\right) \mathcal{H}_{0}^{\prime}\left(b^{*}\right)}{h^{\prime}\left(b^{*}\right) \mathcal{H}_{2 \gamma}^{\prime}\left(b^{*}\right)+h_{2 \gamma}^{\prime}\left(b^{*}\right) \mathcal{H}_{0}^{\prime}\left(b^{*}\right)} \\
D & =\frac{r_{\gamma}}{s_{\gamma}-r_{\gamma}} \frac{\gamma}{\gamma+\delta} \frac{1}{s_{\gamma}} \frac{h_{0}^{\prime \prime}\left(b^{*}\right) \mathcal{H}_{2 \gamma}^{\prime}\left(b^{*}\right)-h_{2 \gamma}^{\prime \prime}\left(b^{*}\right) \mathcal{H}_{0}^{\prime}\left(b^{*}\right)}{h_{0}^{\prime}\left(b^{*}\right) \mathcal{H}_{2 \gamma}^{\prime}\left(b^{*}\right)+h_{2 \gamma}^{\prime}\left(b^{*}\right) \mathcal{H}_{0}^{\prime}\left(b^{*}\right)}
\end{aligned}
$$

where the functions $\mathcal{H}_{0}(\xi)$ and $\mathcal{H}_{2 \gamma}(\xi)$ are defined as

$$
\begin{gathered}
\mathcal{H}_{0}(\xi)=h_{0}^{\prime}(\xi)-s_{\gamma}\left(1-\frac{\gamma}{\gamma+\delta}\right) h_{0}(\xi) \\
\mathcal{H}_{2 \gamma}(\xi)=h_{2 \gamma}^{\prime}(\xi)-s_{\gamma}\left(1+\frac{\gamma}{\gamma+\delta}\right) h_{2 \gamma}(\xi)
\end{gathered}
$$

It is remarkable that the exact same functional form of $\mathcal{H}_{0}(\xi)$ in (5.23) also appeared in Albrecher, Gerber, and Shiu (2011b, see, e.g., denominator of Equation (19)) with Erlang(1) inter-decision times. The two functions $\mathcal{H}_{0}(\xi)$ and $\mathcal{H}_{2 \gamma}(\xi)$ are important building blocks to obtain the analytical properties of $G\left(x, 1 ; b^{*}\right)$ and $G\left(x, 2 ; b^{*}\right)$.

Now substituting coefficients $C$ and $D$ from (5.21) and (5.22) into (5.18) yields the following equation (see Appendix B for more detail) that the optimal barrier $b^{*}$ satisfies

$$
\frac{h_{0}^{\prime \prime}\left(b^{*}\right)}{\mathcal{H}_{0}^{\prime}\left(b^{*}\right)}-\frac{h_{2 \gamma}^{\prime \prime}\left(b^{*}\right)}{\mathcal{H}_{2 \gamma}^{\prime}\left(b^{*}\right)}=2 \frac{s_{\gamma}-r_{\gamma}}{s_{\gamma}} \frac{\gamma+\delta}{\gamma} .
$$

Here (5.25) is a key equation to solve for the optimal periodic barrier $b^{*}$. This is a generalisation to Equation (21) in Albrecher, Gerber, and Shiu (2011b), which solves for the optimal periodic barrier with Erlang(1) inter-decision times. Furthermore, (5.25) also enables us to study the signs of coefficient $A, B, C$ and $D$ and subsequently determine analytical properties of the functions $G\left(x, 2 ; b^{*}\right)$ and $G\left(x, 1 ; b^{*}\right)$.

Remark 5.2. Unfortunately there is no explicit solution for $b^{*}$ from (5.25) since the equation contains a combination of four different exponential terms. Nevertheless one can easily find a solution for (5.25) using numerical methods. A strictly positive solution may not exist under some combinations of parameters (as illustrated in Figure 3 on page 22). Indeed, other cases include a zero barrier ( $\left.b^{*}=0\right)$, which is shown to be optimal in Section 4 , and negative barriers $\left(b^{*}<0\right.$, strategies are not admissible). As explained earlier, due to the lack of an explicit form of (5.25), we cannot conclude that conditions where $b^{*}$ does not exists are equivalent to conditions when a liquidation-at-first-strategy is optimal. But we can partially support this claim using numerical examples in Section 6.3. 
Lemma 5.1. The two functions defined in (5.23) and (5.24) can be re-expressed in the following way,

$$
\begin{aligned}
\mathcal{H}_{0}(\xi) & =s_{\gamma} \frac{\gamma}{\gamma+\delta}\left(\frac{r_{0} e^{r_{0} \xi}}{s_{\gamma}-s_{0}}-\frac{s_{0} e^{s_{0} \xi}}{s_{\gamma}-r_{0}}\right) ; \\
\mathcal{H}_{2 \gamma}(\xi) & =-s_{\gamma} \frac{\gamma}{\gamma+\delta}\left(\frac{r_{2 \gamma} e^{r_{2 \gamma} \xi}}{s_{\gamma}-s_{2 \gamma}}-\frac{s_{2 \gamma} e^{s_{2 \gamma} \xi}}{s_{\gamma}-r_{2 \gamma}}\right) .
\end{aligned}
$$

Using the two function forms, we can obtain an alternative and useful form of (5.25)

$$
s_{\gamma}\left(1-\frac{\gamma}{\gamma+\delta}\right) \frac{\mathcal{H}_{0}\left(b^{*}\right)}{\mathcal{H}_{0}^{\prime}\left(b^{*}\right)}+s_{\gamma}\left(1+\frac{\gamma}{\gamma+\delta}\right) \frac{\mathcal{H}_{2 \gamma}\left(b^{*}\right)}{\mathcal{H}_{2 \gamma}^{\prime}\left(b^{*}\right)}=2 .
$$

We now focus on the signs of $A, B, C$, and $D$. Using the first part of Lemma 5.1 (see Appendix C for a proof) and the ordering of the roots $s_{2 \gamma}<s_{\gamma}<s_{0}<0<r_{0}<r_{2 \gamma}$, we can show that $\mathcal{H}_{0}(\xi)$ is a strictly positive and convex function (i.e. $\mathcal{H}_{0}(\xi)>0$ and $\mathcal{H}_{0}^{\prime \prime}(\xi)>0$ ) and that $\mathcal{H}_{2 \gamma}(\xi)$ is a strictly positive and increasing function (i.e. $\mathcal{H}_{2 \gamma}(\xi)>0$ and $\left.\mathcal{H}_{2 \gamma}^{\prime}(\xi)>0\right)$ for all $\xi \in(0, \infty)$. This subsequently lead to $\mathcal{H}_{0}\left(b^{*}\right)$, $\mathcal{H}_{2 \gamma}\left(b^{*}\right)$ and $\mathcal{H}_{2 \gamma}^{\prime}\left(b^{*}\right)$ being all strictly positive. As a result, inequality $\mathcal{H}_{0}^{\prime}\left(b^{*}\right)>0$ leads to the L.H.S. of (5.28) being negative (contradicting the R.H.S being 2). In summary, we have the following useful results

$$
\mathcal{H}_{0}\left(b^{*}\right)>0, \quad \mathcal{H}_{0}^{\prime}\left(b^{*}\right)<0, \quad \mathcal{H}_{2 \gamma}\left(b^{*}\right)>0, \quad \mathcal{H}_{2 \gamma}^{\prime}\left(b^{*}\right)>0 .
$$

Note that the above results only hold true when the optimal barrier $b^{*}>0$ is applied. These results are used in Section 5.2 to analyse properties of functions $G\left(x, 1 ; b^{*}\right)$ and $G\left(x, 2 ; b^{*}\right)$.

Remark 5.3. The optimal barrier with a periodic strategy of Erlang(2) inter-decision times (denoted as $b^{*}=b_{(2, \gamma)}^{*}$ ) can be determined using (5.25). On the other hand, in a periodic strategy of Erlang (1) interdecision times, the optimal barrier (denoted as $b_{(1, \gamma)}^{*}$ to be different) satisfies the following equation (see Equation (21) in Albrecher, Gerber, and Shiu, 2011b),

$$
\mathcal{H}_{0}^{\prime}\left(b_{(1, \gamma)}^{*}\right)=h_{0}^{\prime \prime}\left(b_{(1, \gamma)}^{*}\right)-s_{\gamma}\left(1-\frac{\gamma}{\gamma+\delta}\right) h_{0}^{\prime}\left(b_{(1, \gamma)}^{*}\right)=0
$$

Using results from Lemma 5.1 and that $\mathcal{H}_{0}(\xi)$ is a convex function, we have the following inequality,

$$
\mathcal{H}_{0}^{\prime}\left(b_{(2, \gamma)}^{*}\right)<0=\mathcal{H}_{0}^{\prime}\left(b_{(1, \gamma)}^{*}\right) \Longrightarrow b_{(2, \gamma)}^{*}<b_{(1, \gamma)}^{*}
$$

This result is intuitively correct. As dividend decisions are made less frequently (i.e. small $n$ while everything else being held the same), the optimal barrier is lower to allow for more dividends to be issued.

Using the first and second order continuities at $b^{*}$ for $G(x, 1), G_{L}\left(b^{*}, 1\right)=G_{U}\left(b^{*}, 1\right)$ and $G_{L}^{\prime}\left(b^{*}, 1\right)=$ $G_{U}^{\prime}\left(b^{*}, 1\right)$, we have

$$
\begin{aligned}
\left(1-\frac{\gamma}{\gamma+\delta}\right) A h_{0}\left(b^{*}\right)-\left(1+\frac{\gamma}{\gamma+\delta}\right) B h_{2 \gamma}\left(b^{*}\right) & =\left(\frac{\gamma}{\gamma+\delta}\right) \frac{\mu}{\gamma+\delta}+D \frac{s_{\gamma}-r_{\gamma}}{s_{\gamma} r_{\gamma}} \frac{\gamma+\delta}{\gamma} \\
A h_{0}^{\prime}\left(b^{*}\right)-B h_{2 \gamma}^{\prime}\left(b^{*}\right) & =\left(\frac{\gamma}{\gamma+\delta}\right)+D \frac{s_{\gamma}-r_{\gamma}}{s_{\gamma} r_{\gamma}} \frac{\gamma+\delta}{\gamma} s_{\gamma} .
\end{aligned}
$$

Next we multiply (5.32) by $s_{\gamma}$, subtract the product from (5.33) and substitute $A$ and $B$ from (5.19) and (5.20), and obtain

$$
\frac{\mathcal{H}_{2 \gamma}^{\prime}\left(b^{*}\right) \mathcal{H}_{0}\left(b^{*}\right)-\mathcal{H}_{0}^{\prime}\left(b^{*}\right) \mathcal{H}_{2 \gamma}\left(b^{*}\right)}{h_{0}^{\prime}\left(b^{*}\right) \mathcal{H}_{2 \gamma}^{\prime}\left(b^{*}\right)+h_{2 \gamma}^{\prime}\left(b^{*}\right) \mathcal{H}_{0}^{\prime}\left(b^{*}\right)}=\frac{\gamma}{\gamma+\delta}\left(1-s_{\gamma} \frac{\mu}{\gamma+\delta}\right)>0 .
$$

Using results from (5.29), we establish the following inequalities

$$
\mathcal{H}_{2 \gamma}^{\prime}\left(b^{*}\right) \mathcal{H}_{0}\left(b^{*}\right)-\mathcal{H}_{0}^{\prime}\left(b^{*}\right) \mathcal{H}_{2 \gamma}\left(b^{*}\right)>0 \Longrightarrow h_{0}^{\prime}\left(b^{*}\right) \mathcal{H}_{2 \gamma}^{\prime}\left(b^{*}\right)+h_{2 \gamma}^{\prime}\left(b^{*}\right) \mathcal{H}_{0}^{\prime}\left(b^{*}\right)>0 .
$$


Combining results from (5.29) and (5.35), we can determine the signs of coefficients $A, B$ and $D$,

$$
\begin{aligned}
A & =\frac{\mathcal{H}_{2 \gamma}^{\prime}\left(b^{*}\right)}{h_{0}^{\prime}\left(b^{*}\right) \mathcal{H}_{2 \gamma}^{\prime}\left(b^{*}\right)+h_{2 \gamma}^{\prime}\left(b^{*}\right) \mathcal{H}_{0}^{\prime}\left(b^{*}\right)}>0 ; \\
B & =\frac{\mathcal{H}_{0}^{\prime}\left(b^{*}\right)}{h_{0}^{\prime}\left(b^{*}\right) \mathcal{H}_{2 \gamma}^{\prime}\left(b^{*}\right)+h_{2 \gamma}^{\prime}\left(b^{*}\right) \mathcal{H}_{0}^{\prime}\left(b^{*}\right)}<0 ; \\
D & =\frac{r_{\gamma}}{s_{\gamma}-r_{\gamma}} \frac{\gamma}{\gamma+\delta} \frac{1}{s_{\gamma}} \frac{h_{0}^{\prime \prime}\left(b^{*}\right) \mathcal{H}_{2 \gamma}^{\prime}\left(b^{*}\right)-h_{2 \gamma}^{\prime \prime}\left(b^{*}\right) \mathcal{H}_{0}^{\prime}\left(b^{*}\right)}{h_{0}^{\prime}\left(b^{*}\right) \mathcal{H}_{2 \gamma}^{\prime}\left(b^{*}\right)+h_{2 \gamma}^{\prime}\left(b^{*}\right) \mathcal{H}_{0}^{\prime}\left(b^{*}\right)}=\frac{2 r_{\gamma}}{s_{\gamma}^{2}} \frac{\mathcal{H}_{0}^{\prime}\left(b^{*}\right) \mathcal{H}_{2 \gamma}^{\prime}\left(b^{*}\right)}{h_{0}^{\prime}\left(b^{*}\right) \mathcal{H}_{2 \gamma}^{\prime}\left(b^{*}\right)+h_{2 \gamma}^{\prime}\left(b^{*}\right) \mathcal{H}_{0}^{\prime}\left(b^{*}\right)}<0 .
\end{aligned}
$$

Note that the second equality of (5.38) makes use of the optimality condition (5.25). Then using the fact that $D$ is negative and (5.18), we have

$$
\left(\frac{\gamma}{\gamma+\delta}\right)^{2}+C s_{\gamma}+D=1 \Longleftrightarrow C=\frac{1}{s_{\gamma}}\left(1-\left(\frac{\gamma}{\gamma+\delta}\right)^{2}-D\right)<0 .
$$
$(5.37)$

As a corollary, we can construct the following inequalities using the signs of coefficients in (5.36) and

$$
\begin{aligned}
G_{L}\left(x, 2 ; b^{*}\right) & =\frac{\mathcal{H}_{2 \gamma}^{\prime}\left(b^{*}\right)}{h_{0}^{\prime}\left(b^{*}\right) \mathcal{H}_{2 \gamma}^{\prime}\left(b^{*}\right)+h_{2 \gamma}^{\prime}\left(b^{*}\right) \mathcal{H}_{0}^{\prime}\left(b^{*}\right)} h_{0}(x)+\frac{\mathcal{H}_{0}^{\prime}\left(b^{*}\right)}{h_{0}^{\prime}\left(b^{*}\right) \mathcal{H}_{2 \gamma}^{\prime}\left(b^{*}\right)+h_{2 \gamma}^{\prime}\left(b^{*}\right) \mathcal{H}_{0}^{\prime}\left(b^{*}\right)} h_{2 \gamma}(x) \\
& <\frac{\mathcal{H}_{2 \gamma}^{\prime}\left(b^{*}\right)}{h_{0}^{\prime}\left(b^{*}\right) \mathcal{H}_{2 \gamma}^{\prime}\left(b^{*}\right)+h_{2 \gamma}^{\prime}\left(b^{*}\right) \mathcal{H}_{0}^{\prime}\left(b^{*}\right)} h_{0}(x)-\frac{\mathcal{H}_{0}^{\prime}\left(b^{*}\right)}{h_{0}^{\prime}\left(b^{*}\right) \mathcal{H}_{2 \gamma}^{\prime}\left(b^{*}\right)+h_{2 \gamma}^{\prime}\left(b^{*}\right) \mathcal{H}_{0}^{\prime}\left(b^{*}\right)} h_{2 \gamma}(x) \\
& =G_{L}\left(x, 1 ; b^{*}\right) .
\end{aligned}
$$

This proves the inequality $G_{L}\left(x, 2 ; b^{*}\right)<G_{L}\left(x, 1 ; b^{*}\right)$ for $x \in\left[0, b^{*}\right)$.

In summary the expected present value of dividends until ruin $G\left(x, 1 ; b^{*}\right)$ and $G\left(x, 2 ; b^{*}\right)$ associated with the optimal barrier level $b^{*}$ takes the following forms

$$
\begin{array}{r}
G\left(x, 1 ; b^{*}\right)= \begin{cases}G_{L}\left(x, 1 ; b^{*}\right)=\frac{h_{0}(x) \mathcal{H}_{2 \gamma}^{\prime}\left(b^{*}\right)-h_{2 \gamma}(x) \mathcal{H}_{0}^{\prime}\left(b^{*}\right)}{h_{0}^{\prime}\left(b^{*}\right) \mathcal{H}_{2 \gamma}^{\prime}\left(b^{*}\right)+h_{2 \gamma}^{\prime}\left(b^{*}\right) \mathcal{H}_{0}^{\prime}\left(b^{*}\right)} & x \in\left[0, b^{*}\right) \\
G_{U}\left(x, 1 ; b^{*}\right)=\left(\frac{\gamma}{\gamma+\delta}\right)\left(\frac{\mu}{\gamma+\delta}+x-b^{*}+G_{L}\left(b^{*}, 2 ; b^{*}\right)\right)+D \frac{s_{\gamma}-r_{\gamma}}{s_{\gamma} r_{\gamma}} \frac{\delta}{\gamma} e^{s_{\gamma}\left(x-b^{*}\right)} & x \in\left[b^{*}, \infty\right)\end{cases} \\
G\left(x, 2 ; b^{*}\right)= \begin{cases}G_{L}\left(x, 2 ; b^{*}\right)=\frac{h_{0}(x) \mathcal{H}_{2 \gamma}^{\prime}\left(b^{*}\right)+h_{2 \gamma}(x) \mathcal{H}_{0}^{\prime}\left(b^{*}\right)}{h_{0}^{\prime}\left(b^{*}\right) \mathcal{H}_{2 \gamma}^{\prime}\left(b^{*}\right)+h_{2 \gamma}^{\prime}\left(b^{*}\right) \mathcal{H}_{0}^{\prime}\left(b^{*}\right)} & x \in\left[0, b^{*}\right) \\
G_{U}\left(x, 2 ; b^{*}\right)=\left(\frac{\gamma}{\gamma+\delta}\right)^{2}\left(\frac{2 \mu}{\gamma+\delta}+x-b^{*}+G_{L}\left(b^{*}, 2 ; b^{*}\right)\right)+\left(C+D\left(x-b^{*}\right)\right) e^{s_{\gamma}\left(x-b^{*}\right)} & x \in\left[b^{*}, \infty\right)\end{cases}
\end{array}
$$

where coefficients $C$ and $D$ can be found in (5.21) and (5.22), and where the optimal barrier $b^{*}>0$ satisfies the following equation

$$
\frac{h_{0}^{\prime \prime}\left(b^{*}\right)}{\mathcal{H}_{0}^{\prime}\left(b^{*}\right)}-\frac{h_{2 \gamma}^{\prime \prime}\left(b^{*}\right)}{\mathcal{H}_{2 \gamma}^{\prime}\left(b^{*}\right)}=2 \frac{s_{\gamma}-r_{\gamma}}{s_{\gamma}} \frac{\gamma+\delta}{\gamma} .
$$

\subsection{Properties of $G\left(x, 1 ; b^{*}\right)$ and $G\left(x, 2 ; b^{*}\right)$}

In this section, we obtain some analytical properties of the functions $G\left(x, 1 ; b^{*}\right)$ and $G\left(x, 2 ; b^{*}\right)$ derived in Section 5.1. First we show that the function $G\left(x, 1 ; b^{*}\right)$ associated with the optimal barrier $b^{*}$ is increasing and concave. We consider the upper branch and obtain the first and second derivatives of $G_{U}\left(x, 1 ; b^{*}\right)$. Using the fact that $D$ is negative from (5.38), it follows that

$$
\begin{aligned}
G_{U}^{\prime}\left(x, 1 ; b^{*}\right) & =\frac{\gamma}{\gamma+\delta}+D \frac{s_{\gamma}-r_{\gamma}}{s_{\gamma} r_{\gamma}} \frac{\gamma+\delta}{\gamma} s_{\gamma} e^{s_{\gamma}\left(x-b^{*}\right)}>0 \\
G_{U}^{\prime \prime}\left(x, 1 ; b^{*}\right) & =D \frac{s_{\gamma}-r_{\gamma}}{s_{\gamma} r_{\gamma}} \frac{\gamma+\delta}{\gamma} s_{\gamma}^{2} e^{s_{\gamma}\left(x-b^{*}\right)}<0
\end{aligned}
$$


for $x \in\left[b^{*}, \infty\right)$. This proves that $G_{U}\left(x, 1 ; b^{*}\right)$ is an increasing and concave function. Next we obtain the first derivative of $G_{L}\left(x, 1 ; b^{*}\right)$

$$
G_{L}^{\prime}\left(x, 1 ; b^{*}\right)=\frac{h_{0}^{\prime}(x) \mathcal{H}_{2 \gamma}^{\prime}\left(b^{*}\right)-h_{2 \gamma}^{\prime}(x) \mathcal{H}_{0}^{\prime}\left(b^{*}\right)}{h_{0}^{\prime}\left(b^{*}\right) \mathcal{H}_{2 \gamma}^{\prime}\left(b^{*}\right)+h_{2 \gamma}^{\prime}\left(b^{*}\right) \mathcal{H}_{0}^{\prime}\left(b^{*}\right)} .
$$

Using the facts that $h_{0}^{\prime}(x)$ and $h_{2 \gamma}^{\prime}(x)$ are both positive and using the signs of the coefficients $A$ and $B$ from (5.36) and (5.37), it follows that $G_{L}^{\prime}\left(x, 1 ; b^{*}\right)>0$ for $x \in\left[0, b^{*}\right)$. To show that the second derivative $G_{L}^{\prime \prime}\left(x, 1 ; b^{*}\right)$ is negative, we obtain the third derivative of $G_{L}\left(x, 1 ; b^{*}\right)$,

$$
G_{L}^{\prime \prime \prime}\left(x, 1 ; b^{*}\right)=\frac{h_{0}^{\prime \prime \prime}(x) \mathcal{H}_{2 \gamma}^{\prime}\left(b^{*}\right)-h_{2 \gamma}^{\prime \prime \prime}(x) \mathcal{H}_{0}^{\prime}\left(b^{*}\right)}{h_{0}^{\prime}\left(b^{*}\right) \mathcal{H}_{2 \gamma}^{\prime}\left(b^{*}\right)+h_{2 \gamma}^{\prime}\left(b^{*}\right) \mathcal{H}_{0}^{\prime}\left(b^{*}\right)}
$$

which is positive (using a similar argument to that used to show that $G_{L}^{\prime}\left(x, 1 ; b^{*}\right)>0$ ). This means that $G_{L}^{\prime \prime}\left(x, 1 ; b^{*}\right)$ is an increasing function. Furthermore we know that $G_{L}^{\prime \prime}\left(b^{*}, 1 ; b^{*}\right)=G_{U}^{\prime \prime}\left(b^{*}, 1 ; b^{*}\right)<0$, which shows that $G_{L}^{\prime \prime}\left(x, 1 ; b^{*}\right)<0$ for $x \in\left[0, b^{*}\right)$. This completes the proof that $G\left(x, 1 ; b^{*}\right)$ is an increasing and concave function.

Now we consider the function $G\left(x, 2 ; b^{*}\right)$ and obtain the first derivative of $G_{U}\left(x, 2 ; b^{*}\right)$

$$
\begin{aligned}
G_{U}^{\prime}\left(x, 2 ; b^{*}\right) & =\left(\frac{\gamma}{\gamma+\delta}\right)^{2}+\left(D+C s_{\gamma}+D\left(x-b^{*}\right) s_{\gamma}\right) e^{s_{\gamma}\left(x-b^{*}\right)} \\
& =\left(\frac{\gamma}{\gamma+\delta}\right)^{2}+\left(1-\left(\frac{\gamma}{\gamma+\delta}\right)^{2}+D\left(x-b^{*}\right) s_{\gamma}\right) e^{s_{\gamma}\left(x-b^{*}\right)}
\end{aligned}
$$

which is positive since $D$ is negative. This shows that $G_{U}^{\prime}\left(x, 2 ; b^{*}\right)>0$ for $x \in\left[b^{*}, \infty\right)$. Next we obtain the second derivative $G_{U}^{\prime \prime}\left(x, 2 ; b^{*}\right)$,

$$
G_{U}^{\prime \prime}\left(x, 2 ; b^{*}\right)=\left(D+1-\left(\frac{\gamma}{\gamma+\delta}\right)^{2}+D\left(x-b^{*}\right) s_{\gamma}\right) s_{\gamma} e^{s_{\gamma}\left(x-b^{*}\right)}, \quad x \in\left[b^{*}, \infty\right) .
$$

To show that $G_{U}^{\prime}\left(x, 2 ; b^{*}\right)$ is bounded, we show that $G_{U}^{\prime}\left(x, 2 ; b^{*}\right)$ attains a finite maximum. So we first solve the equation $G_{U}^{\prime \prime}\left(x, 2 ; b^{*}\right)=0$ for $x \in\left[b^{*}, \infty\right)$,

$$
\begin{aligned}
& \left(D+1-\left(\frac{\gamma}{\gamma+\delta}\right)^{2}+D\left(x-b^{*}\right) s_{\gamma}\right) s_{\gamma} e^{s_{\gamma}\left(x-b^{*}\right)}=0 \\
\Longrightarrow & D+1-\left(\frac{\gamma}{\gamma+\delta}\right)^{2}+D\left(x-b^{*}\right) s_{\gamma}=0 .
\end{aligned}
$$

There are two possibilities that can occur here. First assume that $D+1-\left(\frac{\gamma}{\gamma+\delta}\right)^{2}>0$ and since $D$ is negative, we have

$$
D+1-\left(\frac{\gamma}{\gamma+\delta}\right)^{2}+D\left(x-b^{*}\right) s_{\gamma}>0
$$

which means that $G_{U}^{\prime \prime}\left(x, 2 ; b^{*}\right)<0$. Then in this case the derivative is bounded by $G_{U}^{\prime}\left(x, 2 ; b^{*}\right)<G_{U}^{\prime}\left(b^{*}, 2 ; b^{*}\right)=$ 1. On the other hand, we consider the second possibility $D+1-\left(\frac{\gamma}{\gamma+\delta}\right)^{2}<0$. Observe that $(5.49)$ is linear and increasing in $x$, then there exists a finite point $x_{0} \in\left[b^{*}, \infty\right)$ (because $D$ is finite) such that

$$
D+1-\left(\frac{\gamma}{\gamma+\delta}\right)^{2}+D\left(x_{0}-b^{*}\right) s_{\gamma}=0
$$


This means that $G_{U}^{\prime \prime}\left(x, 2 ; b^{*}\right)>0$ for $x \in\left[b^{*}, x_{0}\right)$ and $G_{U}^{\prime \prime}\left(x, 2 ; b^{*}\right)<0$ for $x \in\left[x_{0}, \infty\right)$ and therefore $x_{0}$ achieves the maximum for $G_{U}^{\prime}\left(x, 2 ; b^{*}\right)$. Then we arrive at the inequality $G_{U}^{\prime}\left(x, 2 ; b^{*}\right)<G_{U}^{\prime}\left(x_{0}, 2 ; b^{*}\right)<\infty$ for all $x \in\left[b^{*}, \infty\right)$. As a result, we are able to show that $G_{U}\left(x, 2 ; b^{*}\right)$ has bounded derivative for $x \in\left[b^{*}, \infty\right)$ in either scenario. In the case of the lower branch $G_{L}\left(x, 2 ; b^{*}\right)$, we have shown that $G_{L}\left(x, 2 ; b^{*}\right)$ is bounded by an increasing and concave function $G_{L}\left(x, 1 ; b^{*}\right)$ for $x \in\left[0, b^{*}\right]$ (see Equation (5.40)). Next since the derivative of $G_{L}^{\prime}\left(x, 2 ; b^{*}\right)$ is a continuous function on a closed interval $\left[0, b^{*}\right]$, then it is bounded on that interval.

In summary, the two functions $G\left(x, 1 ; b^{*}\right)$ and $G\left(x, 2 ; b^{*}\right)$ are continuous and bounded above by some linear functions with properties summarised in the table as follows. These properties will be used to verify the global optimality of periodic barrier strategies with Erlang(2) inter-decision times.

\begin{tabular}{|c|c|c|}
\hline & $x \in\left[0, b^{*}\right)$ & $x \in\left[b^{*}, \infty\right)$ \\
\hline$G\left(x, 1 ; b^{*}\right)$ & increasing and concave & increasing and concave \\
$G\left(x, 2 ; b^{*}\right)$ & $0<G\left(x, 2 ; b^{*}\right)<G\left(x, 1 ; b^{*}\right)$ with bounded derivative & increasing with bounded derivative \\
\hline
\end{tabular}

Table 1: Analytical properties $G\left(x, 1 ; b^{*}\right)$ and $G\left(x, 2 ; b^{*}\right)$

\subsection{Global optimality of the periodic barrier strategies with Erlang(2) inter-decision times}

In this section, we verify that periodic barrier strategies are indeed globally optimal, when inter-dividend decisions times are Erlang $(2)$ distributed. We take the explicit solutions of $G\left(x, 1 ; b^{*}\right)$ and $G\left(x, 2 ; b^{*}\right)$ derived in Section 5.1 with the analytical properties summarised in Table 1 above. Next we substitute the two function $G\left(x, 1 ; b^{*}\right)$ and $G\left(x, 2 ; b^{*}\right)$ into the verification lemma and demonstrate that

$$
G\left(x, 1 ; b^{*}\right)=V(x ; 1) \quad \text { and } \quad G\left(x, 2 ; b^{*}\right)=V(x ; 2) .
$$

We begin with (3.3) and have

$$
e^{-\delta(t \wedge \tau)} G\left(X(t \wedge \tau), \epsilon(t \wedge \tau) ; b^{*}\right)=\sum_{i=1}^{2} e^{-\delta(t \wedge \tau)} G\left(X(t \wedge \tau), i ; b^{*}\right) \eta_{i}(t \wedge \tau) .
$$

After the same simplifications used in Lemma 3.1, for $i=1$ or 2 , we have

$$
\begin{aligned}
& \mathbb{E}_{x, i}\left[e^{-\delta(t \wedge \tau)} G\left(X(t \wedge \tau), \epsilon(t \wedge \tau) ; b^{*}\right)\right]=G\left(x, i ; b^{*}\right) \\
& +\mathbb{E}_{x, i}\left[\int_{0}^{t \wedge \tau} \sigma e^{-\delta s} G^{\prime}\left(X(s-), 1 ; b^{*}\right) \eta_{1}(s) d W(s)\right]+\mathbb{E}_{x, i}\left[\int_{0}^{t \wedge \tau} \sigma e^{-\delta s} G^{\prime}\left(X(s-), 2 ; b^{*}\right) \eta_{2}(s) d W(s)\right] \\
& +\mathbb{E}_{x, i}\left[\int_{0}^{t \wedge \tau} e^{-\delta s}\left(G\left(X(s-), 1 ; b^{*}\right)-G\left(X(s-), 2 ; b^{*}\right)\right) \eta_{2}(s-) d \tilde{N}_{\gamma}(s)\right] \\
& +\mathbb{E}_{x, i}\left[\int_{0}^{t \wedge \tau} e^{-\delta s}\left(\xi_{s}^{*}+G\left(X(s-)-\xi_{s}^{*}, 2 ; b^{*}\right)-G\left(X(s-), 1 ; b^{*}\right)\right) \eta_{1}(s-) d \tilde{N}_{\gamma}(s)\right] \\
& -\mathbb{E}_{x, i}\left[\int_{0}^{t \wedge \tau} e^{-\delta s} \xi_{s}^{*} \eta_{2}(s) d N_{\gamma}(s)\right]
\end{aligned}
$$


Next we show that the following 4 stochastic integrals are uniformly integrable martingales and hence have zero expectations,

$$
\begin{aligned}
& M_{1}(t)=\int_{0}^{t} \sigma e^{-\delta s} G^{\prime}\left(X(s-), 1 ; b^{*}\right) \eta_{1}(s) d W(s) \\
& M_{2}(t)=\int_{0}^{t} \sigma e^{-\delta s} G^{\prime}\left(X(s-), 2 ; b^{*}\right) \eta_{2}(s) d W(s) \\
& M_{3}(t)=\int_{0}^{t} e^{-\delta s}\left(G\left(X(s-), 1 ; b^{*}\right)-G\left(X(s-), 2 ; b^{*}\right)\right) \eta_{2}(s-) d \tilde{N}_{\gamma}(s) \\
& M_{4}(t)=\int_{0}^{t \wedge \tau} e^{-\delta s}\left(\xi_{s}^{*}+G\left(X(s-)-\xi_{s}^{*}, 2 ; b^{*}\right)-G\left(X(s-), 1 ; b^{*}\right)\right) \eta_{1}(s-) d \tilde{N}_{\gamma}(s) .
\end{aligned}
$$

We start with the stochastic integrals $M_{1}(t)$ and $M_{2}(t)$. First since we know that $G\left(x, 1 ; b^{*}\right)$ is increasing and concave, therefore $G^{\prime}\left(x, 1 ; b^{*}\right)$ is bounded above by a finite number, $G^{\prime}\left(x, 1 ; b^{*}\right) \leq G^{\prime}\left(0,1 ; b^{*}\right)<\infty$ for $x \in[0, \infty)$. We also establish in Section 5.2 that $G\left(x, 2 ; b^{*}\right)$ has bounded first derivative, so we can construct the following inequalities

$$
\begin{array}{r}
\mathbb{E}_{x, i}\left[\int_{0}^{t}\left[\sigma e^{-\delta s} G^{\prime}\left(X(s-), 1 ; b^{*}\right) \eta_{1}(s)\right]^{2} d s\right] \leq \mathbb{E}_{x, i}\left[\int_{0}^{t}\left[\sigma e^{-\delta s} G^{\prime}\left(0,1 ; b^{*}\right)\right]^{2} d s\right]<\infty \\
\mathbb{E}_{x, i}\left[\int_{0}^{t}\left[\sigma e^{-\delta s} G^{\prime}\left(X(s-), 2 ; b^{*}\right) \eta_{2}(s)\right]^{2} d s\right] \leq c \mathbb{E}_{x, i}\left[\int_{0}^{t}\left(\sigma e^{-\delta s}\right)^{2} d s\right]<\infty
\end{array}
$$

for some finite constant $c$. This shows that both $M_{1}(t)$ and $M_{2}(t)$ are uniformly integrable martingales (see, e.g., Theorem 8.27 and Corollary 7.8 in Klebaner, 2005).

Next to show that the stochastic integral $M_{3}(t)$ is a uniformly integrable martingale, we need to verify the following inequality (see Theorems 8.27 and 8.32 in Klebaner, 2005)

$$
\mathbb{E}_{x, i}\left[\int_{0}^{t}\left(e^{-\delta s}\left(G\left(X(s-), 1 ; b^{*}\right)-G\left(X(s-), 2 ; b^{*}\right)\right) \eta_{2}(s-)\right)^{2} \gamma d s\right]<\infty .
$$

Using the fact that $G\left(x, 1 ; b^{*}\right)$ is an increasing and concave function and bounded above by a linear equation, we construct the following inequality

$$
(G(X(s-), 1)-G(X(s-), 2)) \eta_{2}(s-) \leq G(X(s-), 1) \leq a_{1} X(s)+a_{2} \leq a_{1} U(s)+a_{2},
$$

for some finite constants $a_{1}$ and $a_{2}$, and where $U(s)$ is the original surplus process defined in (2.1). Substituting the above inequality into (5.64) yields

$$
\begin{aligned}
& \mathbb{E}_{x, i}\left[\int_{0}^{t}\left(e^{-\delta s}\left(G\left(X(s-), 1 ; b^{*}\right)-G\left(X(s-), 2 ; b^{*}\right)\right) \eta_{2}(s-)\right)^{2} \gamma d s\right] \\
\leq & \mathbb{E}_{x, i}\left[\int_{0}^{t} e^{-2 \delta s}\left(a_{1} U(s)+a_{2}\right)^{2} \gamma d s\right]
\end{aligned}
$$

which is finite by evaluating the integral and expectation. This completes the proof that $M_{3}(t)$ is a uniformly integrable martingale. Finally we verify that $M_{4}(t)$ is a uniformly integrable martingale. We need to show that

$$
\mathbb{E}_{x, i}\left[\int_{0}^{t}\left(e^{-\delta s}\left(\xi_{s}^{*}+G\left(X(s-)-\xi_{s}^{*}, 2 ; b^{*}\right)-G\left(X(s-), 1 ; b^{*}\right)\right) \eta_{1}(s-)\right)^{2} \gamma d s\right]<\infty .
$$


We construct the following inequality

$$
\begin{aligned}
\left(\xi_{s}^{*}+G\left(X(s-)-\xi_{s}^{*}, 2 ; b^{*}\right)-G\left(X(s-), 1 ; b^{*}\right)\right) \eta_{1}(s-) & \leq \xi_{s}^{*}+G\left(X(s-)-\xi_{s}^{*}, 2 ; b^{*}\right)-G\left(X(s-), 1 ; b^{*}\right) \\
& <\xi_{s}^{*}+G\left(X(s-)-\xi_{s}^{*}, 2 ; b^{*}\right) \\
& \leq a_{3} X(s)+a_{4} \\
& \leq a_{3} U(s)+a_{4}
\end{aligned}
$$

for some finite constants $a_{3}$ and $a_{4}$. Here (5.69) uses the fact that $\eta_{1}(s) \leq 1,(5.70)$ follows by removing $G\left(x, 1 ; b^{*}\right)$ from the inequality, and $(5.71)$ uses the facts that $\xi_{s}^{*}<X(s)$ and that $G\left(X(s-)-\xi_{s}^{*}, 2 ; b^{*}\right)$ is bounded above by a linear function. Then the inequality (5.68) becomes

$$
\begin{aligned}
& \mathbb{E}_{x, i}\left[\int_{0}^{t}\left(e^{-\delta s}\left(\xi_{s}^{*}+G\left(X(s-)-\xi_{s}^{*}, 2 ; b^{*}\right)-G\left(X(s-), 1 ; b^{*}\right)\right) \eta_{1}(s-)\right)^{2} \gamma d s\right] \\
\leq & \mathbb{E}_{x, i}\left[\int_{0}^{t} e^{-2 \delta s}\left(a_{3} U(s)+a_{4}\right)^{2} \gamma d s\right],
\end{aligned}
$$

which is finite by evaluating the integral and expectation. This proves that $M_{4}(t)$ is a uniformly integrable martingale. Substituting the four martingales into (5.57) yields

$$
\mathbb{E}_{x, i}\left[e^{-\delta(t \wedge \tau)} G\left(X(t \wedge \tau), \epsilon(t \wedge \tau) ; b^{*}\right)\right]=G\left(x, i ; b^{*}\right)-\mathbb{E}_{x, i}\left[\int_{0}^{t \wedge \tau} e^{-\delta s} \xi_{s}^{*} \eta_{2}(s) d N_{\gamma}(s)\right]
$$

Finally to complete the proof, we will show that

$$
\lim _{t \rightarrow \infty} \mathbb{E}_{x, i}\left[e^{-\delta(t \wedge \tau)} G\left(X(t \wedge \tau), \epsilon(t \wedge \tau) ; b^{*}\right)\right]=0
$$

Remember that the probability of ultimate ruin is 1 ; see Section 2.3. Next, we verify (5.76) by applying the dominated convergence theorem, which requires to identify an integrable random variable $R$ such that $e^{-\delta(t \wedge \tau)} G\left(X(t \wedge \tau), \epsilon(t \wedge \tau) ; b^{*}\right)<R$ where $\mathbb{E}[R]<\infty$. When taking $t \rightarrow \infty$ in (5.75), we only need to consider the case when $\tau$ is finite and by the monotone convergence theorem and (5.76), we have

$$
\begin{aligned}
\lim _{t \rightarrow \infty} \mathbb{E}_{x, i}\left[e^{-\delta(t \wedge \tau)} G\left(X(t \wedge \tau), \epsilon(t \wedge \tau) ; b^{*}\right)\right] & =G\left(x, i ; b^{*}\right)-\lim _{t \rightarrow \infty} \mathbb{E}_{x, i}\left[\int_{0}^{t \wedge \tau} e^{-\delta s} \xi_{s}^{*} \eta_{2}(s) d N_{\gamma}(s)\right] \\
\Longrightarrow & G\left(x, i ; b^{*}\right)=\mathbb{E}_{x, i}\left[\int_{0}^{\tau} e^{-\delta s} \xi_{s}^{*} \eta_{2}(s) d N_{\gamma}(s)\right]=V(x, i)
\end{aligned}
$$

for $i=1$ or 2 . This completes the proof and verifies that the proposed periodic barrier strategies with their associated functions $G\left(x, 1 ; b^{*}\right)$ and $G\left(x, 2 ; b^{*}\right)$ attains the expected present value of dividends of the optimal periodic barrier strategies with Erlang $(2)$ inter-decision times.

\section{Numerical examples}

\subsection{Dominance of the optimal barriers $b^{*}$}

In Section 5, we showed that the optimal periodic strategy with Erlang(2) inter-decision times is of barrier type and that the optimal barrier $b^{*}$ can be found by solving (5.25). Interestingly, this optimal barrier not only attains the maximum for the value of the strategy $G(x, 2 ; b)$, but also attains the maximum for the auxiliary function $G(x, 1 ; b)$. We illustrate this result here and set $\mu=1$ and $\sigma=1$, force of interest $\delta=0.1$, and dividend frequency $\gamma=0.1$. Given the above parameters, we obtain the expected present value of dividends $G(x, 2 ; b)$ and $G(x, 1 ; b)$ for arbitrary barrier levels $b$ and also determine the optimal barrier $b^{*}$.

In Figure 2, we plot the expected present value of dividends until ruin for periodic barrier strategies with Erlang $(2)$ inter-decision times (i.e. $G(x, 1 ; b)$ and $G(x, 2 ; b))$. The surface plots of the expected present 


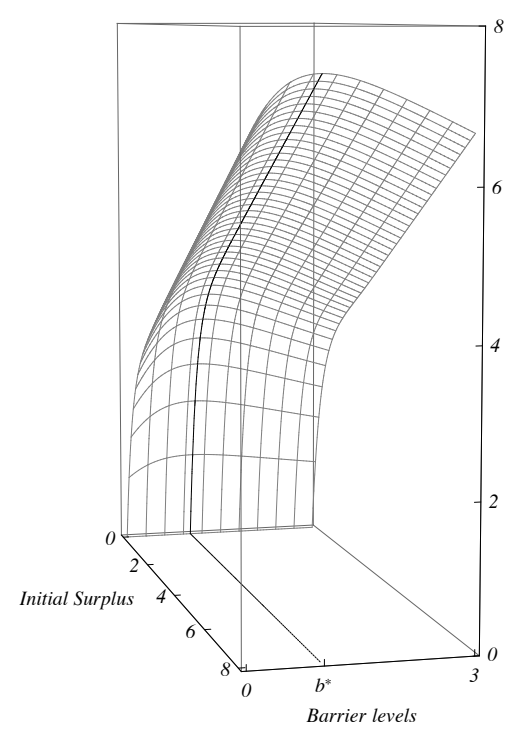

(a) Surface plot for $G(x, 1 ; b)$

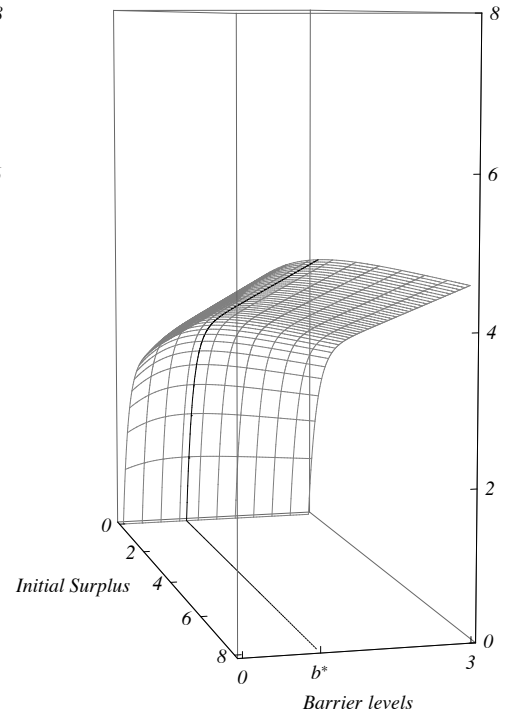

(b) Surface plot for $G(x, 2 ; b)$

Figure 2: Optimal/non-optimal barrier strategies with $\operatorname{Erlang}(2)$ inter-dividend-decision times

value of dividends are produced as a function of two variables: initial surplus $(x)$ and barrier level $(b)$. We first observe that as the number of clock ticks remaining decreases, the expected present value of dividends increases for all values of $x$ and $b$ (e.g., $G(x, 1 ; b)>G(x, 2 ; b)$ illustrated in Figure 2). This makes sense as the time until next dividend decision decreases (with all other parameters held equal), the expected present value of dividends increases due to the time value of money. Next we observe that the optimal barrier $b^{*}$ (outlined by the black line) achieves the maximum for all values of $x$ and across all functions for all $x$ in Figure 2.

\subsection{Impact of parameters on the optimal barrier}

In Section 5.1, we obtain a key equation (5.25) that determines the optimal barrier $b^{*}>0$ in a periodic barrier strategy with Erlang(2) inter-decision times. Since the explicit solution for $b^{*}$ is not available, we illustrate the existence of $b^{*}$ using numerical illustrations. We find the optimal barriers $b^{*}$ using (5.25) while changing the following four parameters: drift $(\mu)$, volatility $(\sigma)$, financial impatience $(\delta)$ and dividend frequency $(\gamma)$. In the following four figures, we change one parameter while keeping others constant. For example in Figure 3(a), the drift parameter changes from 0 to 5 while keeping other parameters constant $(\sigma=1, \delta=0.1, \gamma=0.1)$. Parameters that yield non-positive optimal barrier $b^{*}$ are illustrated in grey regions.

In Figure 3(a), we can observe that when the drift $(\mu)$ is small, the optimal barrier is also small since there is no much expected growth in the surplus. As the drift increases but its magnitude still remains low, the optimal barrier increases to maintain low dividend payouts and ensure that the surplus does not enter ruin. As the drift further increases (e.g. the company has higher growth for identical levels of volatility), the business is safer, and the optimal barrier decreases to allow for more dividends to be paid at each decision time. Next in Figure 3(b) when volatility is very low, the optimal barrier is very small as there is not much risk for the surplus process to ruin. As volatility increases, the optimal barrier increases to maintain the safety of the company. When volatility further increases, the optimal barrier starts to decrease as the company is too volatile to be worth investing into. In Figure 3(c) when the individual becomes more financially impatient (increasing $\delta$ ), more dividends are distributed at each decision time (decreasing $\left.b^{*}\right)$ to compensate for the financial impatience. In Figure 3(d) as the inter-decision times becomes smaller (increasing $\gamma$ ), the optimal barrier increases to allow for smaller but more frequent dividend payments. 


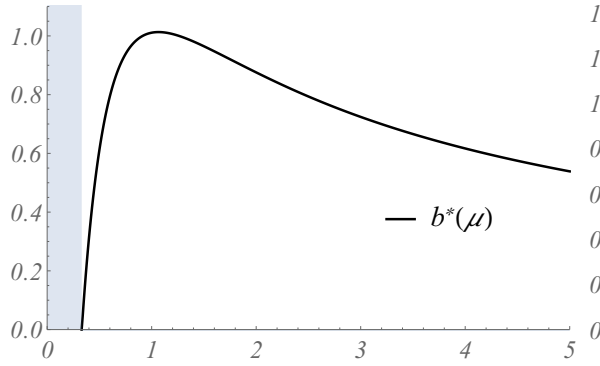

(a) $\mu \in(0,5), \sigma=1, \delta=0.1, \gamma=0.1$

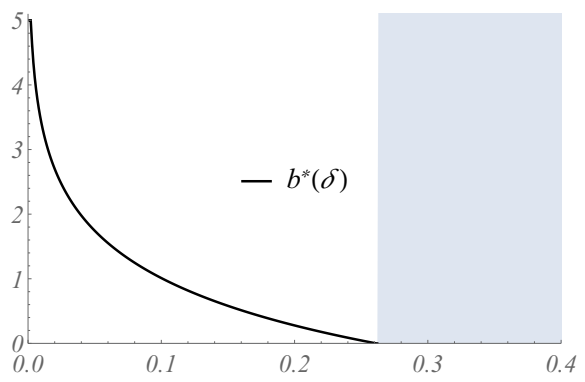

(c) $\mu=1, \sigma=1, \delta \in(0,0.4), \gamma=0.1$

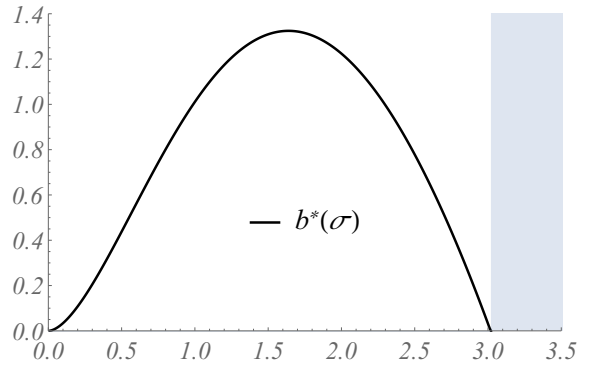

(b) $\mu=1, \sigma \in(0,3.5), \delta=0.1, \gamma=0.1$

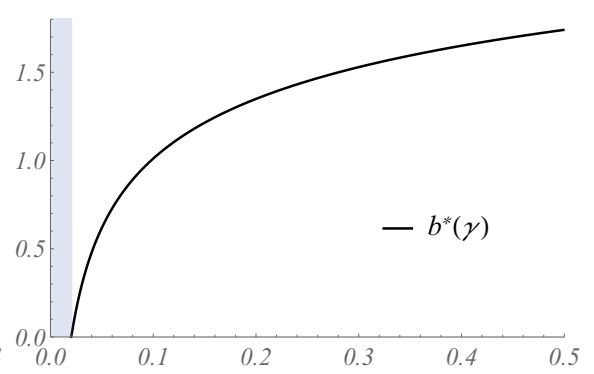

(d) $\mu=1, \sigma=1, \delta=0.1, \gamma \in(0,0.5)$

Figure 3: Impact of different parameters on the optimal barrier $b^{*}$

We further observe that the optimal barriers in each figure become non-positive under certain combinations of the parameter. For example when the drift is really small (low expected growth), the optimal barrier becomes non-positive (grey region in Figure 3(a)). Similarly as the volatility $(\sigma)$ increases, the force of interest $(\delta)$ increases or the dividend frequency $(\gamma)$ decreases. These all represent aggravated prospects, and we postulate that a liquidation-at-first-opportunity is optimal in those regions.

\subsection{The connection between $b^{*}>0$ and $b^{*}=0$}

In Sections 4 and 5, we separately demonstrated the optimality of liquidation-at-first-opportunity strategies $\left(b^{*}=0\right)$ and periodic strategies with strictly positive barriers $\left(b^{*}>0\right)$, given their respective insufficient and aggravated conditions. We are unable to show that the two conditions form perfect complements analytically, but the complementary relationship can be demonstrated through the following numerical illustrations. In Figure 4, we enlarge the grey regions in Figure 3 and plot the optimal barrier $b^{*}$ (black lines) and $F^{\prime}(0,2)$ (derived in Section 4, grey lines) as functions of parameters. We observe that the point at which the optimal barriers reaches zero is the same point as when the equality $F^{\prime}(0,2)=1$ is achieved (illustrated by the dotted line). As prospects worsen (inside the grey region), the inequality $F^{\prime}(0,2) \leq 1$ is satisfied and the liquidation-at-first-opportunity strategy $\left(b^{*}=0\right)$ is optimal.

\section{Conclusion}

An "Erlangisation" technique is typically used to study optimal dividend problems with fixed interdecision times. So far, the literature has mainly focused on the special case of exponential inter-decision times. This is because studying higher dimensional models tends to be very difficult. In this paper, we show that a periodic barrier strategy is still optimal when inter-dividend decision times are Erlang(2) distributed in a Brownian risk model.

We first develop and prove a verification lemma to assess the optimality of any periodic dividend strategy with Erlang inter-dividend decision times, which uses the underlying Markov structure of Erlang periodic dividend strategies. We then use this lemma and show that a liquidation-at-first-opportunity strategy is 


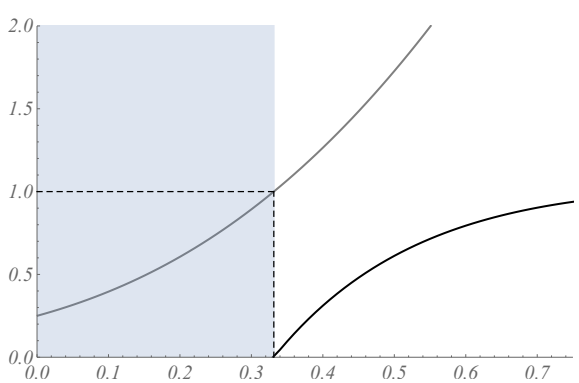

(a) $\mu \in(0,0.75), \sigma=1, \delta=0.1, \gamma=0.1$

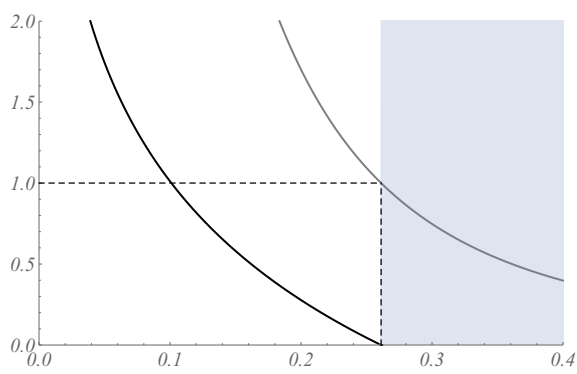

(c) $\mu=1, \sigma=1, \delta \in(0,0.4), \gamma=0.1$

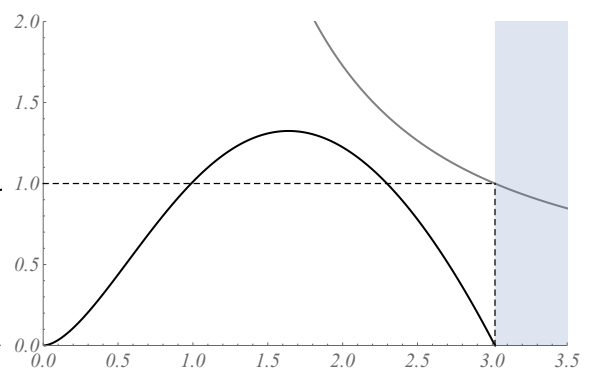

(b) $\mu=1, \sigma \in(0,3.5), \delta=0.1, \gamma=0.1$

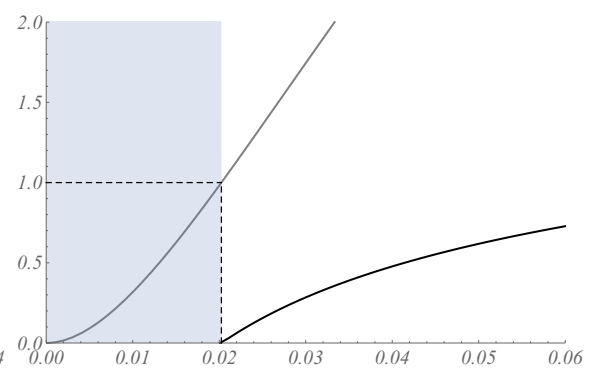

(d) $\mu=1, \sigma=1, \delta=0.1, \gamma \in(0,0.06)$

Figure 4: Impact of different parameters on the optimal barrier $b^{*}$

optimal with Erlang(2) inter-decision times, when there are insufficient prospects. We next focus on periodic dividend strategies with strictly positive barriers. We build on existing algorithms and offer a more direct approach to calculate the expected present value of dividends until ruin, which subsequently allows us to verify the optimality of periodic barrier strategies. Those two cases, as well as their connection, are illustrated in the last section.

\section{Acknowledgements}

The authors are grateful to the referees for providing useful comments. In particular, one referee suggested a more elegant proof for the probability of ruin derived in Section 2.3, which is now included in the paper. This research was supported under Australian Research Council's Linkage Projects funding scheme (project number LP130100723). Vincent Tu acknowledges financial support from an Australian Postgraduate Award and supplementary scholarships provided by the UNSW Australia Business School. The views expressed herein are those of the authors and are not necessarily those of the supporting organisations.

\section{References}

Albrecher, H., Cheung, E. C. K., Thonhauser, S., 2011a. Randomized observation periods for the compound Poisson risk model: dividends. ASTIN Bulletin 41 (2), 645-672.

Albrecher, H., Gerber, H. U., Shiu, E. S. W., 2011b. The optimal dividend barrier in the gamma-omega model. European Actuarial Journal 1 (1), 43-55.

Albrecher, H., Thonhauser, S., 2009. Optimality results for dividend problems in insurance. RACSAM Revista de la Real Academia de Ciencias; Serie A, Mathemáticas 100 (2), 295-320.

Albrecher, H., Thonhauser, S., 2012. On optimal dividend strategies in insurance with a random time horizon. World Scientific, pp. $157-180$.

Asmussen, S., Albrecher, H., 2010. Ruin Probabilities, 2nd Edition. Vol. 14 of Advanced Series on Statistical Science and Applied Probability. World Scientific Singapore.

Asmussen, S., Avram, F., Usabel, M., 2002. Erlangian approximations for finite-horizon ruin probabilities. ASTIN Bulletin $32(2), 267-281$.

Avanzi, B., 2009. Strategies for dividend distribution: A review. North American Actuarial Journal 13 (2), $217-251$. 
Avanzi, B., Cheung, E. C. K., Wong, B., Woo, J.-K., 2013. On a periodic dividend barrier strategy in the dual model with continuous monitoring of solvency. Insurance: Mathematics and Economics 52 (1), 98-113.

Avanzi, B., Tu, V., Wong, B., 2014. On optimal periodic dividend strategies in the dual model with diffusion. Insurance: Mathematics and Economics 55, 210-224.

Avanzi, B., Tu, V. W., Wong, B., 2016. A note on realistic dividends in actuarial surplus models. Risks 4 (4), 37.

Bühlmann, H., 1970. Mathematical Methods in Risk Theory. Grundlehren der mathematischen Wissenschaften. Springer-Verlag, Berlin, Heidelberg, New York.

Choi, M. C., Cheung, E. C. K., 2014. On the expected discounted dividends in the Cramér-Lundberg risk model with more frequent ruin monitoring than dividend decisions. Insurance: Mathematics and Economics 59, 121-132.

de Finetti, B., 1957. Su un'impostazione alternativa della teoria collettiva del rischio. Transactions of the XVth International Congress of Actuaries 2, 433-443.

Gerber, H. U., 1974. The dilemma between dividends and safety and a generalization of the Lundberg-Cramér formulas. Scandinavian Actuarial Journal 1974, 46-57.

Klebaner, F. C., 2005. Introduction to Stochastic Calculus with Applications, 2nd Edition. Imperial College Press, London.

Lipster, R., Shiryaev, A., 1977. Statistics of Random Processes. Springer Verlag, New York, New York.

Pérez, J.-L., Yamazaki, K., 2017. On the optimality of periodic barrier strategies for a spectrally positive Lévy process. Insurance: Mathematics and Economics 77, 1-13.

Wei, J., Wang, R., Yang, H., 2012. On the optimal dividend strategy in a regime-switching diffusion model. Advances in Applied Probability $44(3)$, 886-906.

\section{A. Expected present value of dividends- $\operatorname{Erlang}(n)$ periodic barrier strategies}

In this section, we consider $\operatorname{Erlang}(n)$ periodic strategies with a positive barrier level $b>0$. Under such strategies, dividend payments are $\max \left(X\left(T_{i}\right)-b, 0\right)$ at each dividend decision time $T_{i}$ before ruin. We will derive the expected present value of dividends until ruin by extending and improving a similar algorithm studied in Avanzi, Cheung, Wong, and Woo (2013), where the authors used a probabilistic argument to obtain general solutions for the expected present value of dividends. However the solutions discussed in Avanzi, Cheung, Wong, and Woo (2013) are not explicit enough for an optimality verification. We provide a more direct approach to obtain more explicit solutions of the expected present value of dividends until ruin. This method is used in Section 5.1 to obtain explicit solutions for optimal periodic barrier strategies with Erlang(2) inter-decision times. In the remaining sections of the Appendix, we provide a mathematical formulation of the expected present value of dividends under an arbitrary periodic barrier strategy in Section A.1. We then derive solutions for the expected present value of dividends in Sections A.2 and A.3.

\section{A.1. Model formulation}

First we denote $G(x, i ; b)$ as the expected present value of dividends paid until ruin given that the initial surplus is $x$ and the time remaining until the next dividend payment is distributed as $\operatorname{Erlang}(i)$ for $i=1,2, \ldots, n$. We split the functions $\{G(x, 1 ; b), G(x, 2 ; b), \ldots, G(x, n ; b)\}$ in the following way

$$
G(x, i ; b)= \begin{cases}G_{L}(x, i ; b) & \text { for } x \in[0, b), \\ G_{U}(x, i ; b) & \text { for } x \in[b, \infty),\end{cases}
$$

for $i=1,2, \ldots, n$, with initial conditions $G_{L}(0,1 ; b)=G_{L}(0,2 ; b)=\ldots=G_{L}(0, n ; b)=0$. We further assume that all functions are at least twice differentiable for all $x \geq 0$ and at $x=b$ we have

$$
\lim _{x \rightarrow b} G_{L}(x, i ; b)=G_{U}(b, i ; b) \text { and } \lim _{x \rightarrow b} G_{L}^{\prime}(x, i ; b)=G_{U}^{\prime}(b, i ; b),
$$

for $i=1,2,3, \ldots, n$. The lower and upper branch functions $\left\{G_{L}(x, i ; b)\right\}_{i=1}^{n}$ and $\left\{G_{U}(x, i ; b)\right\}_{i=1}^{n}$ were partially solved in Avanzi, Cheung, Wong, and Woo (2013, Section 3).

\section{A.2. Lower branches}

We first focus on the lower branches and we can show that $G_{L}(x, n ; b), G_{L}(x, n-1 ; b), \ldots, G_{L}(x, 1 ; b)$ satisfy the following system of second order differential equations (see Section 3.1 in Avanzi, Cheung, Wong, 
and Woo, 2013, for a derivation),

$$
\begin{aligned}
(\mathscr{A}-\delta-\gamma) G_{L}(x, n ; b)+\gamma G_{L}(x, n-1 ; b) & =0, \\
(\mathscr{A}-\delta-\gamma) G_{L}(x, n-1 ; b)+\gamma G_{L}(x, n-2 ; b) & =0, \\
\vdots & \\
(\mathscr{A}-\delta-\gamma) G_{L}(x, 2 ; b)+\gamma G_{L}(x, 1 ; b) & =0, \\
(\mathscr{A}-\delta-\gamma) G_{L}(x, 1 ; b)+\gamma G_{L}(x, n ; b) & =0,
\end{aligned}
$$

where the differential operation $\mathscr{A}$ defined in (3.1). We start from (A.3) and make $G_{L}(x, n-1 ; b)$ the subject. Then we can substitute $G_{L}(x, n-1 ; b)$ into (A.4) and make $G_{L}(x, n-2 ; b)$ the subject. We follow this procedure until we get to (A.6) and obtain a $2 n$ degree differential equation for $G_{L}(x, n ; b)$

$$
(\mathscr{A}-\delta-\gamma)^{n} G_{L}(x, n ; b)-(-\gamma)^{n}=0,
$$

with the following characteristic equation,

$$
\left[\frac{\sigma^{2}}{2} \xi^{2}+\mu \xi-(\gamma+\delta)\right]^{n}-(-\gamma)^{n}=0 .
$$

The characteristic equation (A.8) is a $2 n$ degree polynomial with real coefficients, hence yields $2 n$ complex or real roots. To solve (A.8), we consider the equation $\omega^{n}-(-1)^{n}=0$ and find the $n$ roots, $\omega_{1}, \omega_{2}, \ldots, \omega_{n-1}$ and $\omega_{n}$. The $n$ roots are summerised in Table A when $n$ is even or odd. As a further example, we plot the roots for cases $n=7$ and 8 in Figure A.

\begin{tabular}{lccccccc}
\hline & $\omega_{1}$ & $\omega_{2}$ & $\cdots$ & $\cdots$ & $\cdots$ & $\omega_{n-1}$ & $\omega_{n}$ \\
\hline$n$ is odd & $e^{\frac{\pi}{n} i}$ & $e^{\frac{3 \pi}{n} i}$ & $\cdots$ & $e^{\frac{n \pi}{n} i}$ & $\cdots$ & $e^{\frac{(2 n-3) \pi}{n} i}$ & $e^{\frac{(2 n-1) \pi}{n} i}$ \\
$n$ is even & $e^{\frac{2 \pi}{n} i}$ & $e^{\frac{4 \pi}{n} i}$ & $\cdots$ & $e^{\frac{n \pi}{n} i}$ & $\cdots$ & $e^{\frac{(2 n-2) \pi}{n} i}$ & $e^{\frac{2 n \pi}{n} i}$ \\
\hline
\end{tabular}

Table A: Roots to the characteristic equation $\omega_{1}, \ldots, \omega_{n-1}$ and $\omega_{n}$

Next we substitute $\omega_{k}$ back into (A.8) and obtain the following $n$ quadratic equations,

$$
\frac{\sigma^{2}}{2} \xi^{2}+\mu \xi-(\gamma+\delta)=\gamma \omega_{k}, \quad k=1,2, \ldots, n .
$$

Each of those quadratic equations gives two roots $r_{k}$ and $s_{k}$. Now that we have obtained the $2 n$ roots to the characteristic equation (A.8), a general solution for $G_{L}(x, n ; b)$ can be written as

$$
G_{L}(x, n ; b)=\sum_{k=1}^{n}\left(L_{r, k} \cdot e^{r_{k} x}+L_{s, k} \cdot e^{s_{k} x}\right), \quad x \in[0, b),
$$

where $L_{r, k}$ and $L_{s, k}$ are constant coefficients for $k=1,2, \ldots, n$.

The above general solution of $G_{L}(x, n ; b)$ can now be substituted into (A.3) to determine a general solution of $G_{L}(x, n-1 ; b)$. Applying operator $(\mathscr{A}-\delta-\gamma)$ to exponential functions $e^{r_{k} x}$ and $e^{s_{k} x}$ for $k=1,2, \ldots, n$ yields

$$
\begin{aligned}
& (\mathscr{A}-\delta-\gamma)\left(e^{r_{k} x}\right)=\left(\frac{\sigma^{2}}{2} r_{k}^{2}+\mu r_{k}-(\gamma+\delta)\right) e^{r_{k} x}=\gamma \omega_{k} e^{r_{k} x}, \\
& (\mathscr{A}-\delta-\gamma)\left(e^{s_{k} x}\right)=\left(\frac{\sigma^{2}}{2} s_{k}^{2}+\mu s_{k}-(\gamma+\delta)\right) e^{s_{k} x}=\gamma \omega_{k} e^{s_{k} x}
\end{aligned}
$$




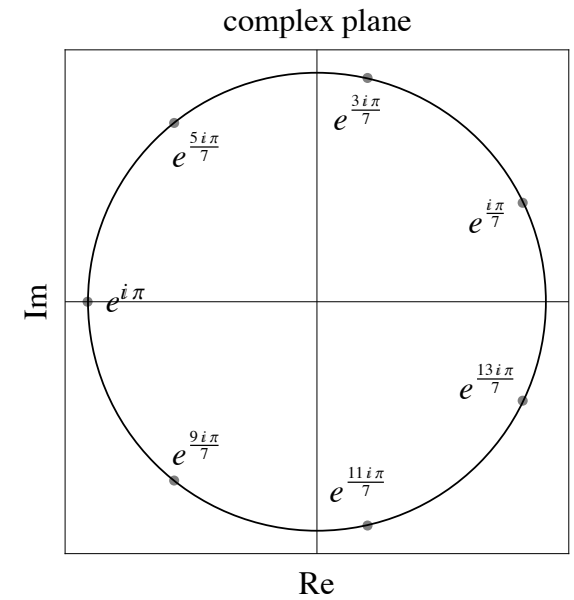

(a) $n=7$

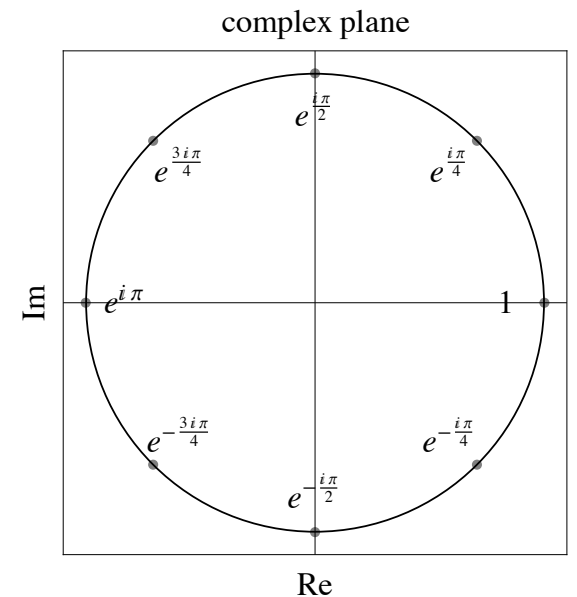

(b) $n=8$

Figure A: Roots to equation $\omega^{n}-(-1)^{n}=0$

Substituting (A.11) and (A.12) into (A.3), we obtain a general solution for $G_{L}(x, n-1 ; b)$

$$
G_{L}(x, n-1 ; b)=\frac{1}{\gamma}(\mathscr{A}-\delta-\gamma) \sum_{k=1}^{n}\left(L_{r, k} \cdot e^{r_{k} x}+L_{s, k} \cdot e^{s_{k} x}\right)=\sum_{k=1}^{n}\left(-\omega_{k}\right)\left(L_{r, k} \cdot e^{r_{k} x}+L_{s, k} \cdot e^{s_{k} x}\right) .
$$

Following this approach, we substitute $G_{L}(x, n-1 ; b)$ into (A.4) to obtain a general solution for $G_{L}(x, n-2 ; b)$ and so on until we finally substitute $G_{L}(x, 2 ; b)$ into (A.5). General solutions for all the lower branch functions $G_{L}(x, n ; b), G_{L}(x, n-1 ; b), \ldots, G_{L}(x, 2 ; b)$ and $G_{L}(x, 1 ; b), x \in[0, b)$, are then

$$
\begin{aligned}
G_{L}(x, n ; b) & =\sum_{k=1}^{n}\left(L_{r, k} \cdot e^{r_{k} x}+L_{s, k} \cdot e^{s_{k} x}\right), \\
\vdots & \\
G_{L}(x, 2 ; b) & =\sum_{k=1}^{n}\left(-\omega_{k}\right)^{n-2}\left(L_{r, k} \cdot e^{r_{k} x}+L_{s, k} \cdot e^{s_{k} x}\right), \\
G_{L}(x, 1 ; b) & =\sum_{k=1}^{n}\left(-\omega_{k}\right)^{n-1}\left(L_{r, k} \cdot e^{r_{k} x}+L_{s, k} \cdot e^{s_{k} x}\right) .
\end{aligned}
$$

Using initial conditions $G_{L}(0, n ; b)=G_{L}(0, n-1 ; b)=\ldots=G_{L}(0,2 ; b)=G_{L}(0,1 ; b)=0$ yields,

$$
\sum_{k=1}^{n}\left(L_{r, k}+L_{s, k}\right)=\sum_{k=1}^{n}\left(-\omega_{k}\right)\left(L_{r, k}+L_{s, k}\right)=\cdots=\sum_{k=1}^{n}\left(-\omega_{k}\right)^{n-1}\left(L_{r, k}+L_{s, k}\right)=0,
$$

which has the following implications,

$$
L_{r, 1}+L_{s, 1}=L_{r, 2}+L_{s, 2}=\ldots=L_{r, n-1}+L_{s, n-1}=L_{r, n}+L_{s, n}=0 .
$$

Let $L_{k}=L_{r, k}=-L_{s, k}$ and substituting into (A.14)-(A.16), general solutions for $G_{L}(x, n ; b), G_{L}(x, n-1 ; b)$, 
$\ldots, G_{L}(x, 2 ; b)$ and $G_{L}(x, 1 ; b)$ for $x \in[0, b)$ now become

$$
\begin{aligned}
G_{L}(x, n ; b) & =\sum_{k=1}^{n} L_{k} \cdot\left(e^{r_{k} x}-e^{s_{k} x}\right), \\
\vdots & \\
G_{L}(x, 2 ; b) & =\sum_{k=1}^{n}\left(-\omega_{k}\right)^{n-2} L_{k} \cdot\left(e^{r_{k} x}-e^{s_{k} x}\right), \\
G_{L}(x, 1 ; b) & =\sum_{k=1}^{n}\left(-\omega_{k}\right)^{n-1} L_{k} \cdot\left(e^{r_{k} x}-e^{s_{k} x}\right) .
\end{aligned}
$$

Remark A.1. Note that when solving the system (A.3)-(A.6), we started by identifying a general solution of $G_{L}(x, n ; b)$ and sequentially identifying general solutions for $G_{L}(x, n-1 ; b), \ldots, G_{L}(x, 1 ; b)$. Since the system of equations is cyclic, the solving of the system can start with any function; the rest of the general solutions can sequentially be obtained.

\section{A.3. Upper branches}

Now we obtain general solutions for the upper branches, $G_{U}(x, n ; b), G_{U}(x, n-1 ; b), \ldots, G_{U}(x, 2 ; b)$ and $G_{U}(x, 1 ; b)$. They satisfy the following system of differential equations (see Section 3.1 in Avanzi, Cheung, Wong, and Woo, 2013, for a derivation)

$$
\begin{aligned}
(\mathscr{A}-\delta-\gamma) G_{U}(x, n ; b)+\gamma G_{U}(x, n-1 ; b) & =0 \\
\vdots & \\
(\mathscr{A}-\delta-\gamma) G_{U}(x, 2 ; b)+\gamma G_{U}(x, 1 ; b) & =0 ; \\
(\mathscr{A}-\delta-\gamma) G_{U}(x, 1 ; b)+\gamma\left[x-b+G_{U}(b, n ; b)\right] & =0 .
\end{aligned}
$$

First we note that from (A.22) $G_{U}(x, 1 ; b)$ satisfies a second order inhomogeneous differential equation. Next we substitute (A.21) into (A.22) and this yields a 4th order inhomogeneous differential equation. This procedure can be done sequentially (for $k-1$ times) to show that $G_{U}(x, k ; b)$ satisfies a $2 k$-th order inhomogeneous differential equation for $k=2,3, \ldots, n$. By assuming that $\left\{G_{U}(x, k ; b)\right\}_{k=1}^{n}$ approaches a linear function as $x \rightarrow \infty$ (see Remark 3.2 in Avanzi, Cheung, Wong, and Woo, 2013, for a proof), we can write down general solutions for $G_{L}(x, n ; b), G_{L}(x, n-1 ; b), \ldots, G_{L}(x, 2 ; b)$ and $G_{L}(x, 1 ; b)$,

$$
\begin{aligned}
G_{U}(x, n ; b) & =\phi(x, n)+\left(\sum_{k=0}^{n-1} U_{k, n} \cdot(x-b)^{k}\right) e^{s_{\gamma}(x-b)} \\
\vdots & \\
G_{U}(x, 2 ; b) & =\phi(x, 2)+U_{0,2} \cdot e^{s_{\gamma}(x-b)}+U_{1,2} \cdot(x-b) e^{s_{\gamma}(x-b)} \\
G_{U}(x, 1 ; b) & =\phi(x, 1)+U_{0,1} \cdot e^{s_{\gamma}(x-b)}
\end{aligned}
$$

where $\phi(x, k)$ is a particular solution for $G_{U}(x, k ; b)$ for $k=1,2, \ldots, n$ which takes the form

$$
\phi(x, k)=\left(\frac{\gamma}{\gamma+\delta}\right)^{k}\left(\frac{k \cdot \mu}{\gamma+\delta}+x-b+G_{L}(b, n ; b)\right),
$$

and $U_{i, j}(i=0,1, \ldots, j-1$ and $j=1,2, . ., n)$ are coefficients of homogeneous solutions of $G_{U}(x, n ; b)$, $G_{U}(x, n-1 ; b), \ldots, G_{U}(x, 2 ; b)$ and $G_{U}(x, 1 ; b)$. Note that the index $j$ represents the number of remaining "exponential" clock ticks until the next dividend decision and the index $i$ corresponds to the power in the function $(x-b)^{i} e^{s_{\gamma}(x-b)}$. 
Remark A.2. Equation (A.26) is an updated version of equation (4.7) in Avanzi, Tu, and Wong (2014) and has a nice interpretation when the surplus is large. Suppose that the next dividend decision time will happen in an Erlang(k) distributed amount of time. Also suppose that the current surplus level is very large, so that a dividend is highly likely to be paid at the next dividend decision time. During this period, the surplus is expected to grow $\mu /(\gamma+\delta)$ after each exponential clock tick and reach $k \mu /(\gamma+\delta)+x$ at the next dividend decision time. After a dividend is then paid, the surplus resets to the barrier level $b$ and future prospects have value of $G_{L}(b, n ; b)$. Finally the entire quantity is discounted by a factor of $(\gamma / \gamma+\delta)^{k}$ since the next dividend decision is Erlang(k) away.

We can now substitute the general solutions (A.23)-(A.25) into the system of differential equations (A.20)-(A.22) to obtain the following relationships between all the coefficients. For $j=2,3, \ldots, n$, we have

$$
U_{j-2, j-1}=\frac{s_{\gamma}-r_{\gamma}}{s_{\gamma} r_{\gamma}} \frac{\gamma+\delta}{\gamma}(j-1) U_{j-1, j}
$$

and for $i=0,1,2, \ldots, k-3$, we have

$$
U_{i, k-1}=-\frac{1}{\gamma} \frac{\sigma^{2}}{2}(i+2)(i+1) U_{i+2, k}+\frac{s_{\gamma}-r_{\gamma}}{s_{\gamma} r_{\gamma}} \frac{\gamma+\delta}{\gamma}(i+1) U_{i+1, k}
$$

Note that using the above recursive relationship, we can express all the coefficients in terms of $n$ coefficients $U_{0, n}, U_{1, n}, \ldots, U_{n-1, n}$. Next we prove the above algorithm of fully specifying the upper branch solutions of $G_{U}(x, 1 ; b), G_{U}(x, 2 ; b), \ldots, G_{U}(x, n ; b)$. We start with a general solution for $G_{U}(x, k ; b)$ for $k=2,3, \ldots, n$,

$$
G_{U}(x, k ; b)=\phi(x, k)+\left(\sum_{i=0}^{k-1} U_{i, k} \cdot(x-b)^{i}\right) e^{s_{\gamma}(x-b)},
$$

which consists of a particular solution $\phi(x, k)$ and a homogeneous solution. The main idea is to use the relationship of $(\mathscr{A}-\delta-\gamma) G_{U}(x, k ; b)+\gamma \cdot G_{U}(x, k-1 ; b)=0$ to determine the relationship between the coefficients of of $G_{U}(x, k ; b)$ and $G_{U}(x, k-1 ; b)$. First we will show that the particular solution satisfies the following relationship

$$
(\mathscr{A}-\delta-\gamma) \phi(x, k)+\gamma \cdot \phi(x, k-1)=0,
$$

for $k=2,3, \ldots, n-1, n$. We start by applying the operator $-\frac{1}{\gamma}(\mathscr{A}-\delta-\gamma)$ to $\phi(x, k)$,

$$
-\frac{1}{\gamma}(\mathscr{A}-\delta-\gamma) \phi(x, k)=-\frac{1}{\gamma}(\mathscr{A}-\delta-\gamma)\left[\left(\frac{\gamma}{\gamma+\delta}\right)^{k}\left(\frac{k \cdot \mu}{\gamma+\delta}+x-b+G_{L}(b, n ; b)\right)\right]=\phi(x, k-1) .
$$

Next we apply the operator $-\frac{1}{\gamma}(\mathscr{A}-\delta-\gamma)$ to the sum of exponential functions in (A.29) and we have

$$
\begin{aligned}
& -\frac{1}{\gamma}(\mathscr{A}-\delta-\gamma)\left[\left(\sum_{i=0}^{k-1} U_{i, k} \cdot(x-b)^{i}\right) e^{s_{\gamma}(x-b)}\right] \\
& =-\frac{1}{\gamma}\left[\sum_{i=0}^{k-3}\left(\frac{\sigma^{2}}{2} U_{i+2, k} \cdot(i+2)(i+1)+\left(\sigma^{2} s_{\gamma}+\mu\right) U_{i+1, k} \cdot(i+1)\right)(x-b)^{i}\right. \\
& \left.+\left(\sigma^{2} s_{\gamma}+\mu\right) U_{k-1, k} \cdot(k-1)(x-b)^{k-2}\right] e^{s_{\gamma}(x-b)} .
\end{aligned}
$$

Next by comparing the coefficient of $(x-b)^{k-2} e^{s_{\gamma}(x-b)}$, we obtain

$$
-\frac{\sigma^{2} s_{\gamma}+\mu}{\gamma}(k-1) U_{k-1, k}=U_{k-2, k-1}
$$


By comparing the coefficient of $(x-b)^{i} e^{s_{\gamma}(x-b)}$ is, for $i=0,1, \ldots, k-3$, we have

$$
-\frac{1}{\gamma} \frac{\sigma^{2}}{2}(i+2)(i+1) U_{i+2, k}-\frac{\sigma^{2} s_{\gamma}+\mu}{\gamma}(i+1) U_{i+1, k}=U_{i, k-1} \text {. }
$$

Note that we can simplify $-\frac{\sigma^{2} s_{\gamma}+\mu}{\gamma}$ using the following relationship

$$
-\frac{\sigma^{2} s_{\gamma}+\mu}{\gamma}=\frac{s_{\gamma}-r_{\gamma}}{s_{\gamma} r_{\gamma}} \frac{\gamma+\delta}{\gamma}
$$

The recursive algorithm described in (A.27) and (A.28) shares a similar form to Theorem 2 in Avanzi, Cheung, Wong, and Woo (2013). Both algorithms are able to reduce the number of unknown coefficients from $(n+1) \cdot n / 2$ to $n$. Our more explicit algorithm enables a more direct computation of the general solutions. We summarise the general solutions of the upper branches and provide a visual representation of the recursive relationship between all the coefficients in Figure B; see Example A.1.

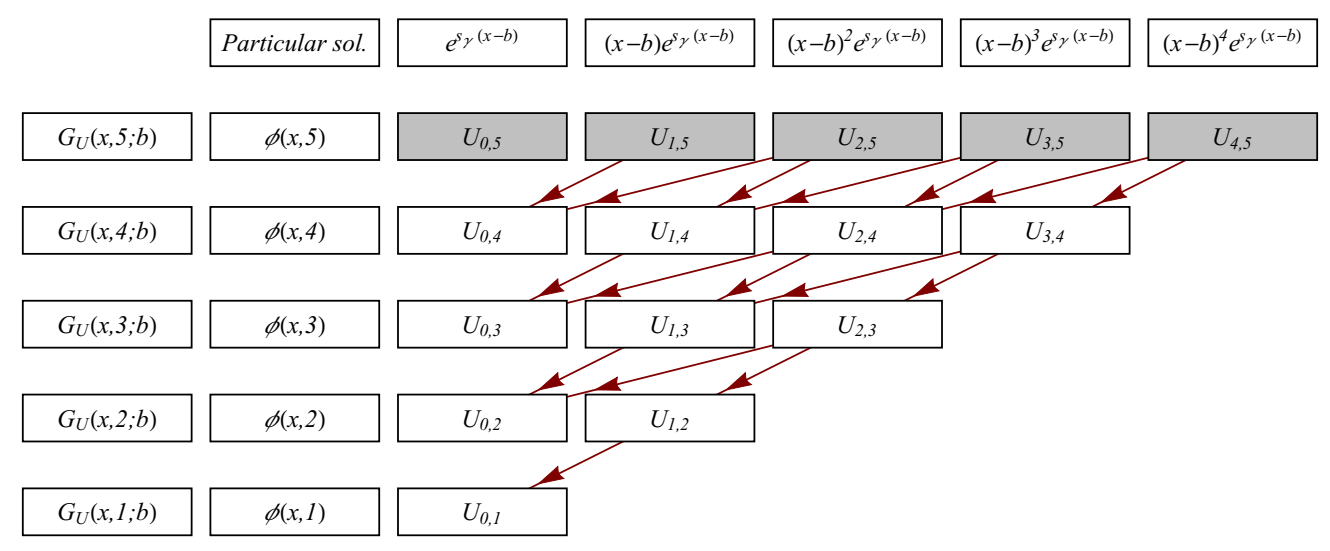

Figure B: General solutions of expected present values of dividends with an Erlang $(5)$ periodic barrier strategy, $\left\{G_{U}(x, k ; b)\right\}_{k=1}^{5}$ consisting of particular and homogeneous solutions

Example A.1. In each row of Figure B, a general solution of $G_{U}(x, k ; b)$ consists of a particular solution $\phi(x, k)$ and a homogeneous solution with coefficients $U_{0, k-1}, U_{1, k-1}, \ldots, U_{k-2, k-1}$. The recursive relationships between the coefficients $U_{i, j}$ for $j=1,2, \ldots, 5$ and $i=0,1, \ldots, j-1$ are summarised using the arrows and as a result all the coefficients can be expressed in terms of $U_{0,5}, \ldots U_{4,5}$ (highlighted in grey cells in Figure $B$ ).

In summary, we now have an easy-to-implement and direct approach to obtain expected present value of dividends under arbitrary periodic barrier strategies with $\operatorname{Erlang}(n)$ inter-decision times $\{G(x, k ; b)\}_{k=1}^{n}$. First we obtain general solutions of $G_{L}(x, 1 ; b), G_{L}(x, 2 ; b), \ldots, G_{L}(x, n ; b)$ from (A.19), which consist of $n$ unknown constants $L_{1}, L_{2}, \ldots, L_{n}$. Next we use a recursive relationship (illustrated in Figure B) and obtain general solutions of $G_{U}(x, 1 ; b), G_{U}(x, 2 ; b), \ldots, G_{U}(x, n ; b)$, which are in terms of $n$ unknown constants $U_{0, n}, U_{1, n}, \ldots, U_{n-1, n}$. In total we have $2 n$ unknown constants we need to solve for. Using the smooth pasting conditions in (A.2), we have $2 n$ equations that are able to determine all the coefficients associated with lower and upper branches $\left\{G_{L}(x, k ; b)\right\}_{k=1}^{n}$ and $\left\{G_{U}(x, k ; b)\right\}_{k=1}^{n}$, respectively.

Remark A.3. For a particular value of $n$, all $n$ functions $G(x, n ; b), \ldots G(x, 1 ; b)$ are determined at the same time (not independently of each other). Furthermore solutions from smaller values of $n$ are updated and not relevant any more, as illustrated in Table $B$. 


\begin{tabular}{cccc}
\hline$n=3$ & & $n=2$ & $n=1$ \\
\hline$G(x, 3 ; b)$ & & & \\
$G(x, 2 ; b)$ & $\neq$ & $G(x, 2 ; b)$ & \\
$G(x, 1 ; b) \neq$ & $\neq$ & \\
\end{tabular}

Table B: Expected present value of dividends until ruin for Erlang(1), (2) and (3) inter-decision times

In Table B, the function $G(x, 2 ; b)$ in the first column (Erlang(3) inter-decision times) is different from the function $G(x, 2 ; b)$ in the second column (Erlang(2) inter-decision times). While both functions correspond to situations where the times until next dividend payment has an Erlang(2) distribution, the prospects once such an Erlang(2) time has elapsed are different, as the next dividend payment will not occur after an identically distributed amount of time.

\section{B. Proof of Equation (5.25)}

We substitute coefficients $C$ and $D$ from (5.22) into (5.18), which yields

$$
\begin{gathered}
\left(\frac{\gamma}{\gamma+\delta}\right)^{2}+C s_{\gamma}+D=1 \Longleftrightarrow s_{\gamma} D+s_{\gamma}^{2} C=s_{\gamma}\left(1-\frac{\gamma}{\gamma+\delta}\right)\left(1+\frac{\gamma}{\gamma+\delta}\right) \\
\Longleftrightarrow \frac{\frac{h_{0}^{\prime \prime}\left(b^{*}\right)}{\mathcal{H}_{0}^{\prime}\left(b^{*}\right)}+\frac{h_{2 \gamma}^{\prime \prime}\left(b^{*}\right)}{\mathcal{H}_{2 \gamma}^{\prime}\left(b^{*}\right)}}{\frac{h_{0}^{\prime}\left(b^{*}\right)}{\mathcal{H}_{0}^{\prime}\left(b^{*}\right)}+\frac{h_{2 \gamma}^{\prime}\left(b^{*}\right)}{\mathcal{H}_{2 \gamma}^{\prime}\left(b^{*}\right)}}-\frac{r_{\gamma}}{s_{\gamma}-r_{\gamma}} \frac{\gamma}{\gamma+\delta} \frac{\frac{h_{0}^{\prime \prime}\left(b^{*}\right)}{\mathcal{H}_{0}^{\prime}\left(b^{*}\right)}-\frac{h_{2 \gamma}^{\prime \prime}\left(b^{*}\right)}{\mathcal{H}_{2 \gamma}^{\prime}\left(b^{*}\right)}}{\frac{h_{0}^{\prime}\left(b^{*}\right)}{\mathcal{H}_{0}^{\prime}\left(b^{*}\right)}+\frac{h_{2 \gamma}^{\prime}\left(b^{*}\right)}{\mathcal{H}_{2 \gamma}^{\prime}\left(b^{*}\right)}}=s_{\gamma}\left(1-\frac{\gamma}{\gamma+\delta}\right)\left(1+\frac{\gamma}{\gamma+\delta}\right) .
\end{gathered}
$$

Then we multiply both hands side by $\frac{h_{0}^{\prime}\left(b^{*}\right)}{\mathcal{H}_{0}^{\prime}\left(b^{*}\right)}+\frac{h_{2 \gamma}^{\prime}\left(b^{*}\right)}{\mathcal{H}_{2 \gamma}^{\prime}\left(b^{*}\right)}$, and obtain

$$
\frac{h_{0}^{\prime \prime}\left(b^{*}\right)}{\mathcal{H}_{0}^{\prime}\left(b^{*}\right)}+\frac{h_{2 \gamma}^{\prime \prime}\left(b^{*}\right)}{\mathcal{H}_{2 \gamma}^{\prime}\left(b^{*}\right)}-\frac{r_{\gamma}}{s_{\gamma}-r_{\gamma}} \frac{\gamma}{\gamma+\delta}\left(\frac{h_{0}^{\prime \prime}\left(b^{*}\right)}{\mathcal{H}_{0}^{\prime}\left(b^{*}\right)}-\frac{h_{2 \gamma}^{\prime \prime}\left(b^{*}\right)}{\mathcal{H}_{2 \gamma}^{\prime}\left(b^{*}\right)}\right)=s_{\gamma}\left(1-\frac{\gamma}{\gamma+\delta}\right)\left(1+\frac{\gamma}{\gamma+\delta}\right)\left(\frac{h_{0}^{\prime}\left(b^{*}\right)}{\mathcal{H}_{0}^{\prime}\left(b^{*}\right)}+\frac{h_{2 \gamma}^{\prime}\left(b^{*}\right)}{\mathcal{H}_{2 \gamma}^{\prime}\left(b^{*}\right)}\right)
$$

After some re-arrangement, we arrive at

$$
\begin{array}{r}
\frac{h_{0}^{\prime \prime}\left(b^{*}\right)}{\mathcal{H}_{0}^{\prime}\left(b^{*}\right)}-s_{\gamma}\left(1-\frac{\gamma}{\gamma+\delta}\right)\left(1+\frac{\gamma}{\gamma+\delta}\right) \frac{h_{0}^{\prime}\left(b^{*}\right)}{\mathcal{H}_{0}^{\prime}\left(b^{*}\right)}+\frac{h_{2 \gamma}^{\prime \prime}\left(b^{*}\right)}{\mathcal{H}_{2 \gamma}^{\prime}\left(b^{*}\right)}-s_{\gamma}\left(1-\frac{\gamma}{\gamma+\delta}\right)\left(1+\frac{\gamma}{\gamma+\delta}\right) \frac{h_{2 \gamma}^{\prime}\left(b^{*}\right)}{\mathcal{H}_{2 \gamma}^{\prime}\left(b^{*}\right)} \\
=\frac{r_{\gamma}}{s_{\gamma}-r_{\gamma}} \frac{\gamma}{\gamma+\delta}\left(\frac{h_{0}^{\prime \prime}\left(b^{*}\right)}{\mathcal{H}_{0}^{\prime}\left(b^{*}\right)}-\frac{h_{2 \gamma}^{\prime \prime}\left(b^{*}\right)}{\mathcal{H}_{2 \gamma}^{\prime}\left(b^{*}\right)}\right)
\end{array}
$$

Next we further re-arrange the above equation and arrive at

$$
\begin{aligned}
& \left(1+\frac{\gamma}{\gamma+\delta}\right) \frac{h_{0}^{\prime \prime}\left(b^{*}\right)-s_{\gamma}\left(1-\frac{\gamma}{\gamma+\delta}\right) h_{0}^{\prime}\left(b^{*}\right)}{\mathcal{H}_{0}^{\prime}\left(b^{*}\right)}-\frac{\gamma}{\gamma+\delta} \frac{h_{0}^{\prime \prime}\left(b^{*}\right)}{\mathcal{H}_{0}^{\prime}\left(b^{*}\right)} \\
+ & \left(1-\frac{\gamma}{\gamma+\delta}\right) \frac{h_{2 \gamma}^{\prime \prime}\left(b^{*}\right)-s_{\gamma}\left(1+\frac{\gamma}{\gamma+\delta}\right) h_{2 \gamma}^{\prime}\left(b^{*}\right)}{\mathcal{H}_{2 \gamma}^{\prime}\left(b^{*}\right)}+\frac{\gamma}{\gamma+\delta} \frac{h_{2 \gamma}^{\prime \prime}\left(b^{*}\right)}{\mathcal{H}_{2 \gamma}^{\prime}\left(b^{*}\right)}=\frac{r_{\gamma}}{s_{\gamma}-r_{\gamma}} \frac{\gamma}{\gamma+\delta}\left(\frac{h_{0}^{\prime \prime}\left(b^{*}\right)}{\mathcal{H}_{0}^{\prime}\left(b^{*}\right)}-\frac{h_{2 \gamma}^{\prime \prime}\left(b^{*}\right)}{\mathcal{H}_{2 \gamma}^{\prime}\left(b^{*}\right)}\right) .
\end{aligned}
$$

Using the functional forms of $\mathcal{H}_{0}^{\prime}\left(b^{*}\right)$ and $\mathcal{H}_{2 \gamma}^{\prime}\left(b^{*}\right)$ in (5.23) and (5.24), we further obtain

$$
\begin{aligned}
& \left(1+\frac{\gamma}{\gamma+\delta}\right)-\frac{\gamma}{\gamma+\delta} \frac{h_{0}^{\prime \prime}\left(b^{*}\right)}{\mathcal{H}_{0}^{\prime}\left(b^{*}\right)}+\left(1-\frac{\gamma}{\gamma+\delta}\right)+\frac{\gamma}{\gamma+\delta} \frac{h_{2 \gamma}^{\prime \prime}\left(b^{*}\right)}{\mathcal{H}_{2 \gamma}^{\prime}\left(b^{*}\right)}=\frac{r_{\gamma}}{s_{\gamma}-r_{\gamma}} \frac{\gamma}{\gamma+\delta}\left(\frac{h_{0}^{\prime \prime}\left(b^{*}\right)}{\mathcal{H}_{0}^{\prime}\left(b^{*}\right)}-\frac{h_{2 \gamma}^{\prime \prime}\left(b^{*}\right)}{\mathcal{H}_{2 \gamma}^{\prime}\left(b^{*}\right)}\right) \\
\Longrightarrow & 2=\frac{r_{\gamma}}{s_{\gamma}-r_{\gamma}} \frac{\gamma}{\gamma+\delta}\left(\frac{h_{0}^{\prime \prime}\left(b^{*}\right)}{\mathcal{H}_{0}^{\prime}\left(b^{*}\right)}-\frac{h_{2 \gamma}^{\prime \prime}\left(b^{*}\right)}{\mathcal{H}_{2 \gamma}^{\prime}\left(b^{*}\right)}\right)+\frac{\gamma}{\gamma+\delta}\left(\frac{h_{0}^{\prime \prime}\left(b^{*}\right)}{\mathcal{H}_{0}^{\prime}\left(b^{*}\right)}-\frac{h_{2 \gamma}^{\prime \prime}\left(b^{*}\right)}{\mathcal{H}_{2 \gamma}^{\prime}\left(b^{*}\right)}\right) \\
\Longrightarrow & \frac{h_{0}^{\prime \prime}\left(b^{*}\right)}{\mathcal{H}_{0}^{\prime}\left(b^{*}\right)}-\frac{h_{2 \gamma}^{\prime \prime}\left(b^{*}\right)}{\mathcal{H}_{2 \gamma}^{\prime}\left(b^{*}\right)}=2 \frac{s_{\gamma}-r_{\gamma}}{s_{\gamma}} \frac{\gamma+\delta}{\gamma},
\end{aligned}
$$


which completes the proof for (5.25).

\section{Proof of Lemma 5.1}

We first provide two identities involving functions $h_{0}(\xi)$ and $h_{2 \gamma}(\xi)$

$$
\begin{aligned}
h_{0}^{\prime}(\xi)-s_{\gamma}\left(1-\frac{\gamma}{\gamma+\delta}\right) h_{0}(\xi) & =\frac{1}{\left(r_{0}+s_{0}\right)-s_{\gamma}}\left(h_{0}^{\prime \prime}(\xi)-s_{\gamma} h_{0}^{\prime}(\xi)\right), \\
h_{2 \gamma}^{\prime}(\xi)-s_{\gamma}\left(1+\frac{\gamma}{\gamma+\delta}\right) h_{2 \gamma}(\xi) & =\frac{1}{\left(r_{2 \gamma}+s_{2 \gamma}\right)-s_{\gamma}}\left(h_{2 \gamma}^{\prime \prime}(\xi)-s_{\gamma} h_{2 \gamma}^{\prime}(\xi)\right),
\end{aligned}
$$

which can be proved by comparing coefficients of exponential terms on both hand sides. Next using the above identities and some relationship of the roots $\left(s_{2 \gamma}, s_{\gamma}, s_{0}, r_{0}, r_{2 \gamma}\right)$, we can establish the following equations for $\mathcal{H}_{0}^{\prime}(\xi)$ and $\mathcal{H}_{2 \gamma}^{\prime}(\xi)$ defined in (5.23) and (5.24),

$$
\begin{aligned}
\mathcal{H}_{0}^{\prime}(\xi) & =\left(r_{0}-s_{0}-s_{\gamma}\right) \mathcal{H}_{0}(\xi)+h_{0}^{\prime}(\xi) s_{\gamma} \frac{\gamma}{\gamma+\delta} \\
\mathcal{H}_{2 \gamma}^{\prime}(\xi) & =\left(r_{2 \gamma}-s_{2 \gamma}-s_{\gamma}\right) \mathcal{H}_{2 \gamma}(\xi)-h_{2 \gamma}^{\prime}(\xi) s_{\gamma} \frac{\gamma}{\gamma+\delta} .
\end{aligned}
$$

By solving the above two first order inhomogeneous differential equations with initial conditions $\mathcal{H}_{0}(0)=$ $r_{0}-s_{0}$ and $\mathcal{H}_{2 \gamma}(0)=r_{2 \gamma}-s_{2 \gamma}$, we obtain alternative forms of $\mathcal{H}_{0}(\xi)$ and $\mathcal{H}_{2 \gamma}(\xi)$

$$
\begin{aligned}
\mathcal{H}_{0}(\xi) & =s_{\gamma} \frac{\gamma}{\gamma+\delta}\left(\frac{r_{0} e^{r_{0} \xi}}{s_{\gamma}-s_{0}}-\frac{s_{0} e^{s_{0} \xi}}{s_{\gamma}-r_{0}}\right) ; \\
\mathcal{H}_{2 \gamma}(\xi) & =-s_{\gamma} \frac{\gamma}{\gamma+\delta}\left(\frac{r_{2 \gamma} e^{r_{2 \gamma} \xi}}{s_{\gamma}-s_{2 \gamma}}-\frac{s_{2 \gamma} e^{s_{2 \gamma} \xi}}{s_{\gamma}-r_{2 \gamma}}\right) .
\end{aligned}
$$

We then use the ordering of the roots $s_{2 \gamma}<s_{\gamma}<s_{0}<0<r_{0}<r_{2 \gamma}$ to show that $\mathcal{H}_{0}(\xi)$ is a strictly positive and convex function (i.e. $\mathcal{H}_{0}(\xi)>0$ and $\mathcal{H}_{0}^{\prime \prime}(\xi)>0$ ) and $\mathcal{H}_{2 \gamma}(\xi)$ is a strictly positive and increasing function (i.e. $\mathcal{H}_{2 \gamma}(\xi)>0$ and $\left.\mathcal{H}_{2 \gamma}^{\prime}(\xi)>0\right)$ for all $\xi \in(0, \infty)$.

Next by making use of the explicit functional forms of $h_{0}^{\prime}(\xi), \mathcal{H}_{0}^{\prime}(\xi), h_{2 \gamma}^{\prime}(\xi)$ and $\mathcal{H}_{2 \gamma}^{\prime}(\xi)$, we can obtain the following two identities

$$
\begin{gathered}
\frac{s_{\gamma}}{s_{\gamma}-r_{\gamma}} \frac{\gamma}{\gamma+\delta} \frac{h_{0}^{\prime}(\xi)}{\mathcal{H}_{0}^{\prime}(\xi)}=\frac{1}{s_{\gamma}-r_{\gamma}}-\frac{r_{\gamma}}{s_{\gamma}-r_{\gamma}} \frac{\mathcal{H}_{0}(\xi)}{\mathcal{H}_{0}^{\prime}(\xi)} \\
-\frac{s_{\gamma}}{s_{\gamma}-r_{\gamma}} \frac{\gamma}{\gamma+\delta} \frac{h_{2 \gamma}^{\prime}(\xi)}{\mathcal{H}_{2 \gamma}^{\prime}(\xi)}=\frac{1}{s_{\gamma}-r_{\gamma}}-\frac{r_{\gamma}}{s_{\gamma}-r_{\gamma}} \frac{\mathcal{H}_{2 \gamma}(\xi)}{\mathcal{H}_{2 \gamma}^{\prime}(\xi)} .
\end{gathered}
$$

Both identities can be shown after some algebra. We begin the proof of (C.5) by substituting the functional 
forms of $h_{0}^{\prime}(\xi)$ and $\mathcal{H}_{0}^{\prime}(\xi)$ into the left hand side of (C.5), which yields

$$
\begin{aligned}
& \frac{s_{\gamma}}{s_{\gamma}-r_{\gamma}} \frac{\gamma}{\gamma+\delta} \frac{h_{0}^{\prime}(\xi)}{\mathcal{H}_{0}^{\prime}(\xi)}=\frac{1}{s_{\gamma}-r_{\gamma}} s_{\gamma} \frac{\gamma}{\gamma+\delta} \frac{h_{0}^{\prime}(\xi)}{\mathcal{H}_{0}^{\prime}(\xi)} \\
& =\frac{\frac{r_{0}}{s_{\gamma}-r_{\gamma}} e^{r_{0} \xi}-\frac{s_{0}}{s_{\gamma}-r_{\gamma}} e^{s_{0} \xi}}{\frac{r_{0}^{2}}{s_{\gamma}-s_{0}} e^{r_{0} \xi}-\frac{s_{0}^{2}}{s_{\gamma}-r_{0}} e^{s_{0} \xi}} \\
& =\frac{\frac{s_{\gamma}-r_{\gamma}+r_{\gamma}-s_{0}}{s_{\gamma}-r_{\gamma}} \frac{r_{0}}{s_{\gamma}-s_{0}} e^{r_{0} \xi}-\frac{s_{\gamma}-r_{\gamma}+r_{\gamma}-r_{0}}{s_{\gamma}-r_{\gamma}} \frac{s_{0}}{s_{\gamma}-r_{0}} e^{s_{0} \xi}}{\frac{r_{0}^{2}}{s_{\gamma}-s_{0}} e^{r_{0} \xi}-\frac{s_{0}^{2}}{s_{\gamma}-r_{0}} e^{s_{0} \xi}} \\
& =\frac{\frac{r_{0}}{s_{\gamma}-s_{0}} e^{r_{0} \xi}+\frac{r_{\gamma}-s_{0}}{s_{\gamma}-r_{\gamma}} \frac{r_{0}}{s_{\gamma}-s_{0}} e^{r_{0} \xi}-\frac{s_{0}}{s_{\gamma}-r_{0}} e^{s_{0} \xi}-\frac{r_{\gamma}-r_{0}}{s_{\gamma}-r_{\gamma}} \frac{s_{0}}{s_{\gamma}-r_{0}} e^{s_{0} \xi}}{\frac{r_{0}^{2}}{s_{\gamma}-s_{0}} e^{r_{0} \xi}-\frac{s_{0}^{2}}{s_{\gamma}-r_{0}} e^{s_{0} \xi}} \\
& =\frac{\frac{r_{0}}{s_{\gamma}-s_{0}} e^{r_{0} \xi}-\frac{s_{0}}{s_{\gamma}-r_{0}} e^{s_{0} \xi}}{\frac{r_{0}^{2}}{s_{\gamma}-s_{0}} e^{r_{0} \xi}-\frac{s_{0}^{2}}{s_{\gamma}-r_{0}} e^{s_{0} \xi}}+\frac{\frac{r_{\gamma}-s_{0}}{s_{\gamma}-r_{\gamma}} \frac{r_{0}}{s_{\gamma}-s_{0}} e^{r_{0} \xi}-\frac{r_{\gamma}-r_{0}}{s_{\gamma}-r_{\gamma}} \frac{s_{0}}{s_{\gamma}-r_{0}} e^{s_{0} \xi}}{\frac{r_{0}^{2}}{s_{\gamma}-s_{0}} e^{r_{0} \xi}-\frac{s_{0}^{2}}{s_{\gamma}-r_{0}} e^{s_{0} \xi}} .
\end{aligned}
$$

Equation (C.6) can be proven in a similar way. Next by substitute the two identities (C.5) and (C.6), into (5.25), we can obtain an alternative and useful form of (5.25)

$$
s_{\gamma}\left(1-\frac{\gamma}{\gamma+\delta}\right) \frac{\mathcal{H}_{0}\left(b^{*}\right)}{\mathcal{H}_{0}^{\prime}\left(b^{*}\right)}+s_{\gamma}\left(1+\frac{\gamma}{\gamma+\delta}\right) \frac{\mathcal{H}_{2 \gamma}\left(b^{*}\right)}{\mathcal{H}_{2 \gamma}^{\prime}\left(b^{*}\right)}=2 .
$$

The proof of Lemma 5.1 is complete. 